\title{
Novel Methods for Approximate Maximum Likelihood Estimation of Multiple Superimposed Undamped Tones and Their Application to Radar Systems
}

\author{
Pasquale Di Viesti, Student Member, IEEE, Alessandro Davoli, Student Member, IEEE, Giorgio Guerzoni and
} Giorgio M. Vitetta, Senior Member, IEEE

\begin{abstract}
In this manuscript, novel methods for the detection of multiple superimposed tones in noise and the estimation of their parameters are derived, and their application to colocated multiple-input multiple-output radar systems is investigated. These methods are based on a maximum likelihood approach and combine an innovative single tone estimator with a serial cancellation procedure. Our numerical results lead to the conclusion that these methods can achieve a substantially better accuracy-complexity tradeoff than various related techniques available in the technical literature. Moreover, they can be exploited to detect multiple closely spaced targets and estimate their spatial coordinates in radar systems.
\end{abstract}

Index terms - Maximum likelihood, frequency estimation, complex exponentials, interpolation algorithm, Fourier coefficients, leakage.

\section{INTRODUCTION}

$\mathbf{T}$ HE problem of estimating the amplitude, phase and frequency of multiple (say, $L$ ) tones in additive white Gaussian noise (AWGN) has received significant attention for a number of years because of its relevance in various fields, including radar systems and wireless communications [1], [2]. It is well known that the maximum-likelihood (ML) approach to this problem leads to a complicated nonlinear optimization problem. Substantial simplifications can be made when the $L$ tone frequencies are sufficiently well separated and the number $N$ of available signal samples is large enough [3]-[5]. In fact, under these assumptions, each tone has a limited influence on the estimation of the others, so that approximate ML estimation can be achieved through a conceptually simple sequential procedure, that consists in iteratively executing two steps [4]. In the first step of this procedure, the parameters of the dominant tone (i.e., of the tone associated with the largest peak in the periodogram of the observed signal) are estimated in a ML fashion. In its second step, instead, the estimated tone is subtracted from the available signal samples and a new periodogram is computed for the resulting residual. These steps are repeated until all the detectable tones are estimated. Note that this simple sequential procedure can be considered as a specific instance of: a) the serial interference cancellation (SIC) concept [6], since, when a new tone is detected and its parameters estimated, all the other tones are considered as a form of interference; b) the so called CLEAN method [7], developed for high-resolution radio interferometry and later used in microwave imaging [8] and radar systems [9], [10]. Moreover, its technical relevance is motivated by the following relevant advantages [11]:

1. It turns a complicated multidimensional problem (whose dimensionality is usually unknown a priori) into a sequence of lower dimensional subproblems. Consequently, its overall complexity is proportional to that required to solve each of such subproblems and is usually much lower than that of: a) parametric estimation methods, like the MUSIC [12], the ESPRIT [13] and their computationally efficient versions (e.g., see [14] and [15]); b) non parametric spectral estimators, like the Capon method [16], the amplitude and phase estimation of a sinusoid (APES) [17], the iterative adaptive approach for amplitude and phase estimation (IAA-APES) [18] and their computationally efficient versions (e.g., see [19] and [20]).

2. It performs better than independently estimating the tones associated with the largest peaks of the original periodogram. In fact, it allows to identify peaks that are initially masked by the leakage due to nearby stronger tones.

3. It is able to estimate an unknown $L$ in a simple fashion. In fact, this result can be achieved setting the initial value of this parameter to zero and applying a suitable test to establish whether, at each repetition of the first step, the largest peak detected in the periodogram of the last residual is significant [3] or whether, at each repetition of the second step, the energy of the new residual is large enough [21]. If one of these conditions is satisfied, the estimate of $L$ is incremented by one and, then, the next step is carried out; otherwise, the estimation process is terminated. It is worth stressing that various estimation methods (e.g., the MUSIC and the ESPRIT estimators) require prior knowledge of $L$ and that, in these cases, the use of some

The authors are with the University of Modena and Reggio Emilia, Dept. of Engineering "Enzo Ferrari", Via P. Vivarelli 10/1, 41125 Modena (Italy) and with Consorzio Nazionale Interuniversitario per le Telecomunicazioni (CNIT), email: pasquale.diviesti@unimore.it, alessandro.davoli@unimore.it, giorgio.guerzoni@unimore.it, giorgio.vitetta@unimore.it. The technical content of this manuscript is patent pending. 
methods, like the generalized Akaike information criterion [22] or the minimum description length [23] (see also refs. [12] and [13]) is commonly proposed for the estimation of this parameter; however, the computational effort they require is not negligible.

The two-step procedure described above, despite its advantages, suffers from the following two shortcomings:

1. Any inaccuracy in the estimation of each single tone accomplished in its first step results in an imperfect cancellation of the tone itself and, consequently, in error accumulation; the intensity of this phenomenon increases with iterations, so affecting the estimation accuracy of the weakest tones.

2. The estimate of each tone is potentially biased, because of the presence of other tones [5]. Biases are influenced by the relative phase, frequency and amplitude of the superimposed tones and are expected to be more relevant in the first estimated frequencies, since these suffer from stronger interference from other tones. For this reason, the overall accuracy of this procedure depends on that of the employed single tone estimator and can be improved by adopting specific methods for mitigating the estimation bias. As far as the first issue is concerned, it is important to point out that optimal (i.e., ML) estimation of a single tone in AWGN is a computationally hard task. This is mainly due to the fact that the ML metric is an highly nonlinear function, that does not lend itself to easy maximisation (e.g., see [24]). In practice, the most accurate ML-based single tone estimators available in the technical literature achieve approximate maximisation of this metric through a two-step procedure; the first step consists in a coarse search of tone frequency, whereas the second one in a fine estimation generating an estimate of the so called frequency residual (i.e., of the difference between the real frequency and its coarse estimate). Coarse estimation is always based on the maximization of the periodogram of the observed signal, whereas fine estimation can be accomplished in an open loop fashion or through an iterative procedure. On the one hand, all the open loop estimators exploit spectral interpolation to infer the frequency residual from the analysis of the fast Fourier transform (FFT) coefficients at the maxima of the associated periodogram and at frequencies adjacent to it [11], [25]-[34]. Unluckily, unlike iterative estimators, the accuracy they achieve is frequency dependent and gets smaller when the signal frequency approaches the center of one of the FFT bins. On the other hand, the iterative estimation techniques available in the technical literature are based on various methods, namely on: a) standard numerical methods for locating the global maximum of a function (e.g., the secan method [35] or the Newton's method [36]); b) an iterative method for binary search, known as the dichotomous search of the periodogram peak [37]-[39]; c) interpolation methods amenable to iterative implementation [40]-[46]; d) the combination of the above mentioned dichotomous search with various interpolation techniques [47]; e) the computation of the first derivative of the spectrum [48].

The use of some of these algorithms in multiple tone estimators based on the above mentioned serial cancellation approach has been investigated in refs. [11], [21], [22], [49], [50] and [51]. More specifically, on the one hand, the periodogram-based (coarse) estimation method has been employed in the CLEAN algorithm [7], in the more CLEAN (MCLEAN) [21] and in the RELAX algorithm [22]. Note that, since a fine estimation step is missing in all these algorithms, achieving high accuracy requires the use of zero padding and of a large FFT order. On the other hand, the exploitation of more refined single tone estimators has been investigated in refs. [11], [49], [50] and [51]. In particular, the use of open-loop interpolation methods exploiting three or five adjacent spectral coefficients (including the one associated with the coarse frequency estimate) has been studied in ref. [11], whereas that of the iterative methods developed in refs. [40] and [52] has been analysed in refs. [49], [50] and [51], respectively.

As far as the second technical issue (i.e., estimation bias) is concerned, it is worth mentioning that the most straightforward methods for bias mitigation rely on the use of a) zero-padding for enhancing periodogram spectral resolution and b) window functions [5], [24], [53]-[56]; the price to be paid for these choices is an increase in the overall computational cost and in the variance of computed estimates, respectively. More refined methods are represented by interpolators with intrinsic leakage rejection [11] and nonlinear optimization methods. The last class of methods includes the expectation maximization (EM) algorithm [57], the space-alternating generalized EM (SAGE) algorithm [58], [59], the Newton's method [36], [60] and different optimization algorithms that employ cyclic cancellation procedures [21], [22], [51], [60]. In the last case, tone re-estimation is accomplished after removing the interference of both stronger and weaker tones as the iterations of the serial cancellation procedure evolve [21], [22] or after detecting and estimating the parameters of all tones [51]; the most refined version of the first method is described in ref. [22], where tone re-estimation is iterated after the estimation of each new tone, in order to generate excellent initial estimates for the next step (i.e., for the estimation of the next tone). Tone re-estimation reduces error accumulation and leads to convergence to the ML solution in the absence of noise if the frequency spacing of the detected tones is large enough; however, this result is achieved at the price of an increase of the overall computational cost and latency [21].

This manuscript aims at providing various new results about the estimation of multiple superimposed tones and their exploitation in colocated frequency modulated continuous wave (FMCW) and stepped frequency continuous wave (SFCW) multiple-input multiple-output (MIMO) radar systems operating at millimeter waves [61], [62]. Its contribution is threefold. First, a novel ML-based iterative estimator of a single real and complex tone is developed. The derivation of this estimator is based on: a) expressing the dependence of the ML metric on the frequency residual in an approximate polynomial form through standard approximations of trigonometric functions; b) exploiting the alternating minimization technique for the maximization of this metric (e.g., see [63, Par. IV-A]). Moreover, its most relevant feature is represented by the fact that it requires the 
evaluation of spectral coefficients that are not exploited by all the other related estimation methods available in the technical literature. Secondly, it is shown how serial cancellation in the frequency domain can be combined with our iterative estimator to detect multiple tones and estimate their parameters. Thirdly, the accuracy of our single and multiple estimators is assessed by extensive computer simulations; both synthetically generated data and the measurements acquired through two commercial MIMO systems in different scenarios are processed. Our results lead to the conclusion that our estimators outperform all the other related estimators in terms of probability of convergence and accuracy in the presence of arbitrary frequency residuals.

The remaining part of this manuscript is organised as follows. In Section II, signal models are defined and their relevance in colocated MIMO radar systems is discussed. Section III is devoted to the derivation of our single tone and multiple tone estimation algorithms, to the assessment of their computational complexity, and to analysis of their similarities and differences with related estimators available in the technical literature. In Section IV, the performance of our estimation algorithms is assessed and compared with that achieved by other estimators. Finally, some conclusions are offered in Section V.

\section{Signal And System Models}

In this manuscript, we focus on the problem of estimating all the parameters of the complex sequence

$$
x_{c, n}=\sum_{l=0}^{L-1} A_{l} \exp \left(j 2 \pi n F_{l}\right)+w_{c, n},
$$

and its real counterpart

$$
\begin{aligned}
x_{r, n} & =\sum_{l=0}^{L-1} \Re\left\{A_{l} \exp \left(j 2 \pi n F_{l}\right)\right\}+w_{r, n} \\
& =\sum_{l=0}^{L-1} a_{l} \cos \left(2 \pi n F_{l}+\psi_{l}\right)+w_{r, n},
\end{aligned}
$$

with $n=0,1, \ldots, N-1$; here, $A_{l}$ and $F_{l} \in[0,1)$ denote the complex amplitude and the normalised frequency, respectively, of the $l$-th complex tone appearing in the right-hand side (RHS) of eq. (1), $a_{l} \triangleq\left|A_{l}\right|, \psi_{l} \triangleq \angle\left(A_{l}\right), w_{c, n}$ is the $n$-th sample of an additive white Gaussian noise (AWGN) sequence (whose elements have zero mean and variance $2 \sigma^{2}$ ), $w_{r, n} \triangleq \Re\left\{w_{c, n}\right\}$, $N$ is the overall number of samples, and $\Re\{x\}$ and $\arg (x)$ denote the real part and the phase, respectively, of the complex quantity $x$. It is useful to point out that the signal model (2) can be rewritten as

$$
x_{r, n}=\sum_{l=0}^{L-1}\left[C_{l} \exp \left(j 2 \pi n F_{l}\right)+C_{l}^{*} \exp \left(-j 2 \pi n F_{l}\right)\right]+w_{r, n},
$$

where

$$
C_{l} \triangleq \frac{1}{2} a_{l} \exp \left(j \psi_{l}\right)=\frac{1}{2} A_{l}
$$

represents the complex amplitude of the real tone appearing in the RHS of eq. (2).

The signal models (1) and (2) appear in a number of problems concerning radar systems, wireless communications and biomedical applications. In the remaining part of this paragraph, we analyse their meaning in the context of colocated and bistatic multiple-input multiple output (MIMO) radar systems operating in time division multiplexing (TDM) [1] and at millimetre waves. These systems have the following important characteristics:

a) They are equipped with a transmit (TX) antenna array and a receive (RX) antenna array, that consist of $N_{T}$ elements and $N_{R}$ elements, respectively; these allow to radiate orthogonal waveforms from different antennas and to receive distinct replicas of the electromagnetic echoes generated by multiple targets.

b) Their TX and RX arrays are made of distinct antenna elements, placed at different positions. Moreover, TX antennas are close to the RX ones and, in particular, are usually placed on the same shield.

c) Orthogonality of the transmitted waveforms is achieved by radiating them through distinct TX antennas over disjoint time intervals.

The architecture of the radar system considered in this manuscript is illustrated in Fig. 1. In the remaining part of this section, two different models are described for the RF signal generated by the voltage controlled oscillator (VCO) of its transmitter and radiated by its TX array after power amplification. In the first case, corresponding to a frequency modulated continuous wave (FMCW) radar system, the VCO is fed by a periodic ramp generator; this produces a chirp FM signal, whose instantaneous frequency evolves periodically, as illustrated in Fig. 2. In this figure, the parameters $T, T_{R}$ and $T_{0}$ represent the chirp interval, the reset time and the pulse period (or pulse repetition interval), respectively [1], whereas the parameters $f_{0}$ and $B$ are the start frequency and the bandwidth, respectively, of the transmitted signal. If we assume that the radar system exploits all the available TX diversity (i.e., all its TX antennas) and that a time slot of $T_{0} \mathrm{~s}$ is assigned to each TX antenna, transmission from the whole TX array is accomplished over an interval lasting $T_{F} \triangleq N_{T} T_{0}$ s; this interval represents the duration of a single 


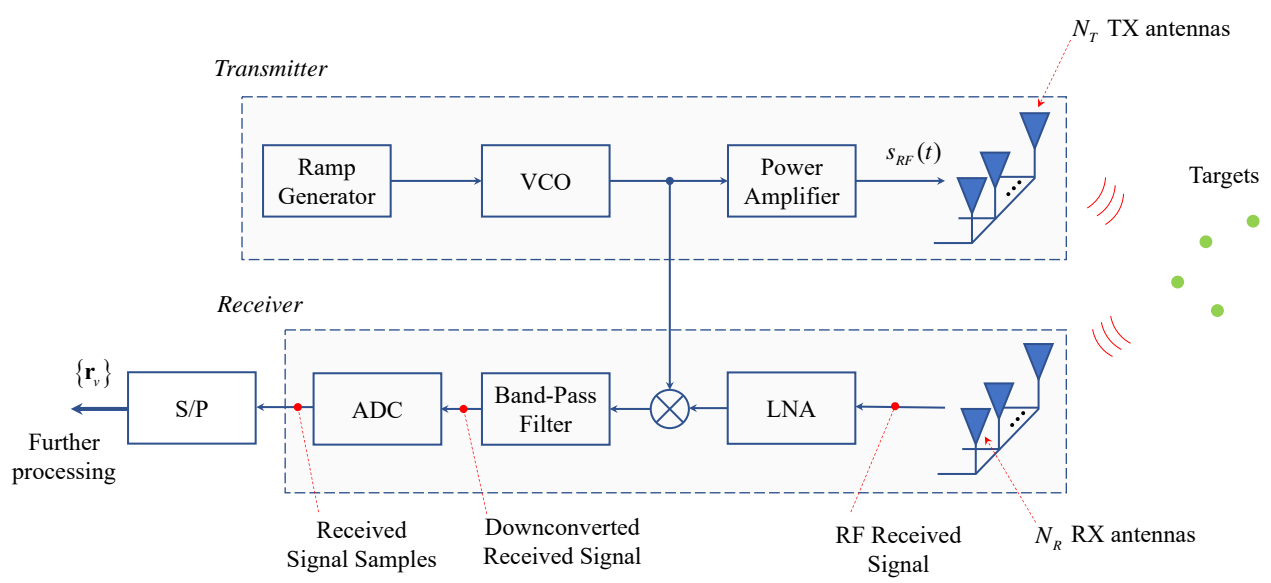

Fig. 1: Transmitter (upper part) and receiver (lower part) of a colocated MIMO radar.

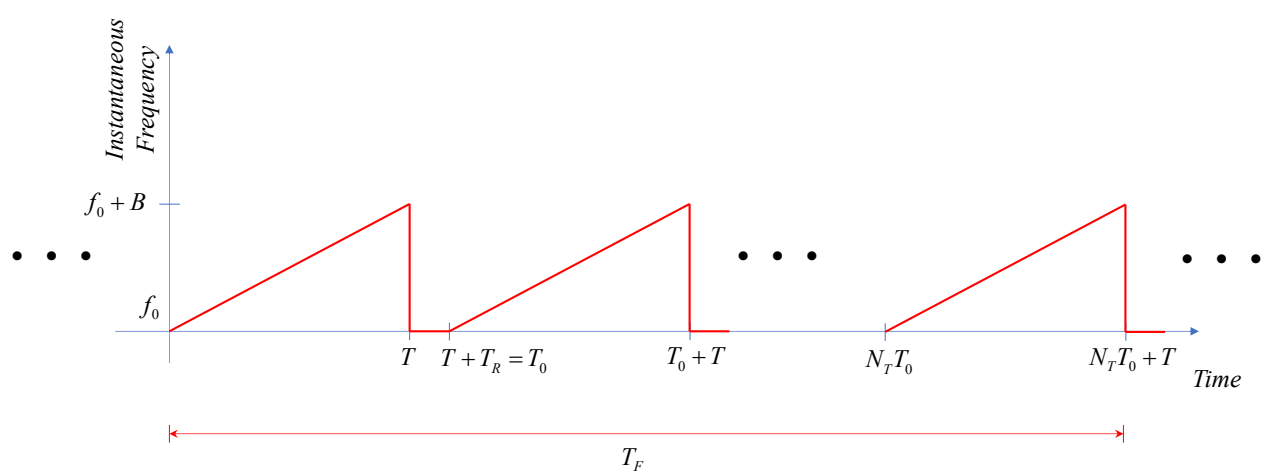

Fig. 2: Representation of the instantaneous frequency of the RF signal generated by the VCO in a FMCW radar system.

transmission frame. Let us focus now on a single chirp interval and, in particular, on the time interval $(0, T)$, and assume that, in that interval, the $p$-th TX antenna is employed by the considered radar system (with $p \in\left\{0,1, \ldots, N_{T}-1\right\}$ ); the signal radiated by that antenna can be expressed as

$$
s_{R F}(t)=A_{R F} \Re\{s(t)\},
$$

where $A_{R F}$ is its amplitude,

$$
\begin{gathered}
s(t) \triangleq \exp (j \theta(t)), \\
\theta(t) \triangleq 2 \pi\left(f_{0} t+\frac{\mu}{2} t^{2}\right)
\end{gathered}
$$

and $\mu \triangleq B / T$ is the chirp rate (i.e., the steepness of the generated frequency chirp). Let $r_{R F}^{(q)}(t)$ denote the signal available at the output of the $q$-th RX antenna in the same time interval, with $q=0,1, \ldots, N_{R}-1$ (see Fig. 1). This signal feeds a low noise amplifier (LNA), whose output undergoes downconversion, filtering and analog-to-digital conversion at a frequency $f_{s}=1 / T_{s}$; here, $T_{s}$ denotes the sampling period of the employed analog-to-digital converter (ADC). If the radiated signal $s_{R F}(t)(5)$ is reflected by $L$ static point targets, the useful component of $r_{R F}^{(q)}(t)$ consists of the superposition of $L$ echoes, each originating from a distinct target. In this case, if the propagation environment is static or undergoes slow variations, a simple mathematical model can be developed to represent the sequence of samples generated by the ADC in a single chirp interval. In deriving that model, the couple of the involved physical TX and RX antennas (namely, the $p$-th TX antenna and the $q$-th $\mathrm{RX}$ antenna) of the considered bistatic radar is usually replaced by a single virtual antenna of an equivalent monostatic radar. In particular, if it is assumed that all the TX and RX antennas belong to the same planar shield and a reference system lying on the physical antenna array is defined, the abscissa $x_{v}$ and the ordinate $y_{v}$ of the $v$-th virtual antenna element associated with the $p$-th TX antenna and the $q$-th RX antenna can be computed as (e.g., see [64, Par. 4.3.1])

$$
x_{v}=\frac{x_{p}+x_{q}}{2}
$$

and

$$
y_{v}=\frac{y_{p}+y_{q}}{2}
$$


respectively, with $v=0,1, \ldots, N_{V R}-1$; here, $\left(x_{p}, y_{p}\right)\left(\left(x_{q}, y_{q}\right)\right)$ are the coordinates of the $\mathrm{TX}\left(\mathrm{RX}\right.$ antenna) and $N_{V R} \triangleq$ $N_{T} \cdot N_{R}$ represents the overall size of the resulting virtual array. Based on these assumptions, the $n$-th received signal sample acquired through the $v$-th virtual antenna element (with $v=0,1, \ldots, N_{V R}-1$ ) can be expressed as (e.g., see [65, Par. 4.6, eq. (4.27)])

$$
x_{r, n}^{(v)}=\sum_{l=0}^{L-1} a_{l} \cos \left(2 \pi n F_{l}^{(v)}+\psi_{l}^{(v)}\right)+w_{r, n}^{(v)},
$$

with $n=0,1, \ldots, N-1$; here, $N$ is the overall number of samples acquired over a chirp period, $a_{l}$ is the amplitude ${ }^{1}$ of the $l$-th component of the useful signal,

$$
F_{l}^{(v)} \triangleq f_{l}^{(v)} T_{s}
$$

is the normalized version of the frequency

$$
f_{l}^{(v)} \triangleq \mu \tau_{l}^{(v)}
$$

characterizing the $l$-th target detected on the $v$-th virtual $\mathrm{RX}$ antenna,

$$
\tau_{l}^{(v)} \triangleq \frac{2}{c}\left[R_{l}+x_{v} \cos \left(\phi_{l}\right) \sin \left(\theta_{l}\right)+y_{v} \sin \left(\phi_{l}\right)\right]
$$

is the delay of the echo generated by the $l$-th target and observed on the $v$-th virtual channel, $R_{l}, \theta_{l}$ and $\phi_{l}$ denote the range of the $l$-th target, its azimuth and its elevation, respectively,

$$
\psi_{l}^{(v)} \cong 2 \pi f_{0} \tau_{l}^{(v)}
$$

and $w_{r, n}^{(v)}$, the $n$-th sample of the AWGN sequence affecting the received signal, is a Gaussian random variable having zero mean and variance $\sigma^{2}$ (assumed to be independent of $v$ ). It is important to point out that: a) the real signal model (10) is identical to that expressed by eq. (2) and can be adopted in all the FMCW radar systems that extract only the in-phase component of the signal captured by each RX antenna; b) some commercial MIMO radar devices provide both the in-phase and quadrature components of the received RF signals (e.g., see [62, Par 2.1 eq. (2.2)]). In the last case, the complex model

$$
x_{c, n}^{(v)}=\sum_{l=0}^{L-1} A_{l}^{(v)} \exp \left(j 2 \pi n F_{l}^{(v)}\right)+w_{c, n}^{(v)},
$$

equivalent to that expressed by eq. (1), must be adopted in place of its real counterpart (10) for any $n$; here,

$$
A_{l}^{(v)} \triangleq a_{l} \exp \left(j \psi_{l}^{(v)}\right)
$$

for any $v$ and $l$.

The second case we consider in the generation of the radiated waveform corresponds to a stepped frequency continuous wave (SFCW) radar system. Its name is motivated by the fact that the VCO of its transmitter is fed by a staircase generator. For this reason, the instantaneous frequency of the resulting RF signal takes on $N$ distinct and uniformly spaced values in an interval lasting $T \mathrm{~s}$ for each TX antenna; the $n$-th value of the instantaneous frequency is

$$
f_{n}=f_{0}+n \Delta f
$$

with $n=0,1, \ldots, N-1$; here, $f_{0}$ is the minimum radiated frequency, $\Delta f$ is the frequency step size and $N$ is the overall number of transmitted frequencies. It can be shown that, under the same assumptions made in the derivation of eq. (15), the measurement acquired through the $v$-th virtual element at the $n$-th frequency can be expressed as

$$
x_{c, n}^{(v)}=\sum_{l=0}^{L-1} A_{l}^{(v)} \exp \left(-j 2 \pi n F_{l}^{(v)}\right)+w_{c, n}^{(v)},
$$

with $v=0,1, \ldots, N_{V R}-1$; here, the amplitude $\psi_{l}^{(v)}$ and the phase $A_{l}^{(v)}$ are still expressed by eqs. (14) and (16), respectively,

$$
F_{l}^{(v)} \triangleq \tau_{l}^{(v)} \Delta f
$$

is the normalised delay characterizing the $l$-th target and observed on the $v$-th virtual antenna, and the parameters $\left(a_{l}\right.$, $\left.\tau_{l}^{(v)}\right)$ and the random variable $w_{c, n}^{(v)}$ have exactly the same meaning as the one illustrated for the received signal model (15).

Then, in both the considered FMCW and SFCW radar systems, the signal observed on each virtual channel can be represented a superposition of $L$ real or complex oscillations; moreover, the value of the parameter $L$ has to be considered unknown. In the

\footnotetext{
${ }^{1}$ This amplitude quantifies the radar cross section (RCS) of the $l$-th target. It depends on both the range and the reflectivity of this target. Moreover, in this manuscript, it is assumed to be independent of the virtual antenna index (i.e., of $v$ ), for simplicity.
} 
following derivations, the real samples $\left\{x_{r, n}^{(v)} ; n=0,1, \ldots, N-1\right\}$ or their complex counterpart $\left\{x_{c, n}^{(v)} ; n=0,1, \ldots, N-1\right\}$ acquired on the $v$-th virtual channel are collected in the $N$-dimensional vector

$$
\mathbf{x}_{z}^{(v)} \triangleq\left[x_{z, 0}^{(v)}, x_{z, 1}^{(v)}, \ldots, x_{z, N-1}^{(v)}\right]^{T}
$$

with $z=r$ or $c$. This vector is processed by the next stages of the radar receiver for target detection and estimation. As it can be easily inferred from eqs. (10)-(12) ((15)-(16) and (18)-(19)), in the considered radar system, the problem of target detection and range estimation on the $v$-th virtual channel is equivalent to the classic problem of detecting multiple overlapped sinusoids (multiple overlapped complex exponentials) in the presence of AWGN and estimating their frequencies [66]. In fact, if, in a FMCW radar system, the $l$-th tone is found at the frequency $\hat{f}_{l}^{(v)}$, the presence of a target at the range (see eqs. (12) and (13))

$$
\hat{R}_{v, l}=\frac{1}{2} \frac{c}{\mu} \hat{f}_{l}^{(v)}
$$

is detected. Similarly, in a SFCW radar system, the normalised delay $\hat{F}_{l}^{(v)}$ estimated on the the $v$-th virtual channel is associated with a target whose range is (see eqs. (13) and (19))

$$
\hat{R}_{v, l}=\frac{1}{2} \frac{c}{\Delta f} \hat{F}_{l}^{(v)}
$$

Information about the angular coordinates (namely, the azimuth and the elevation) of this target, instead, can be acquired through the estimation of the set of $N_{V R}$ phases $\left\{\psi_{l}^{(v)} ; v=0,1, \ldots, N_{V R}-1\right\}$ observed over the available virtual antennas. In fact, since (see eqs. (13) and (14))

$$
\psi_{l}^{(v)} \cong \frac{4 \pi}{\lambda}\left[R_{l}+x_{v} \cos \left(\phi_{l}\right) \sin \left(\theta_{l}\right)+y_{v} \sin \left(\phi_{l}\right)\right],
$$

where

$$
\lambda \triangleq \frac{c}{f_{0}}
$$

is the wavelength associated with the frequency $f_{0}$, the sequence $\left\{\psi_{l}^{(v)} ; v=0,1, \ldots, N_{V R}-1\right\}$ exhibits a periodic behavior characterized by the normalised horizontal spatial frequency

$$
F_{H, l} \triangleq 2 \frac{d_{H}}{\lambda} \cos \left(\phi_{l}\right) \sin \left(\theta_{l}\right),
$$

if the considered virtual elements form an horizontal uniform linear array (ULA), whose adjacent elements are spaced $d_{H}$ $\mathrm{m}$ apart. Dually, if a virtual vertical ULA is assumed, the periodic variations observed in the same sequence of phases are characterized by the normalised vertical spatial frequency

$$
F_{V, l} \triangleq 2 \frac{d_{V}}{\lambda} \sin \left(\phi_{l}\right),
$$

where $d_{V}$ denotes the distance between adjacent elements of the virtual array itself. Consequently, angle finding can be easily accomplished by digital beamforming, i.e. by performing FFT processing on the estimated phases taken across multiple elements of the virtual array in a single frame interval [67], [68].

From the considerations illustrated above, the following conclusions can be easily inferred: a) on the one hand, achieving precise estimation of the range of a given target requires the availability of an accurate estimate of the normalised frequency (or delay) of the real or complex tone associated with the target itself over at least one RX antenna; b) on the other hand, the quality of the estimate of the direction of arrival (DOA) characterizing the radar echo generated by a given target depends on the accuracy of the phase estimated over multiple virtual channels. The last consideration motivates the importance of accurately estimating this parameter over multiple antennas. It is worth stressing that limited attention is often paid to this technical issue in most of the technical literature dealing with the estimation of the parameters of a single tone or multiple superimposed tones; in fact, a lot of emphasis is usually put on the accuracy of frequency estimation, but the quality of phase estimates is neglected (e.g., see [11], [21], [40], [49]-[51]). Finally, in analysing the suitability of multiple tone estimators to colocated MIMO radar systems operating at millimetre waves, the following additional technical issues need to be taken carefully into account:

1) These radar systems often operate at short ranges and in the presence of extended targets. Each of resulting radar images is a cloud of point targets whose mutual spacing can be very small. For this reason, the accuracy of these images depends on the frequency resolution (delay resolution) achieved on each virtual antenna in a FMCW (SFCW) radar system. In fact, this makes the receiver able to separate point targets characterized by similar ranges.

2) Information about the RCS of each point targets can be exploited in the classification of extended targets; for this reason, the availability of an accurate estimate of the amplitude of each radar echo can be very useful in a number of applications (e.g., in SAR imaging [17], [22]). 
3) Distinct radar echoes can be characterized by substantially different signal-to-noise ratios (SNRs).

4) The number $N$ of samples acquired over each virtual channel usually ranges from few hundreds to few thousands.

The last two issues explain why significant attention must be paid to the accuracy achieved by the adopted estimation algorithms at low SNRs and/or for relatively small values of $N$, since this can appreciably influence the quality of the generated radar image.

\section{Approximate Maximum Likelihood Estimation of Single and Multiple Tones}

In this section, we first derive a new method for estimating the parameters of a single real or complex tone. Then, we show how this method can be exploited to detect multiple superimposed tones and estimate their parameters through a deterministic procedure based on successive cancellation. Finally, we analyse the computational complexity of the developed estimation methods and compare them with some related techniques available in the technical literature.

\section{A. Estimation of a single tone}

Let us focus first on the problem of estimating the parameters (namely, the frequency and complex amplitude) of a single tone contained in the real sequence $\left\{x_{r, n} ; n=0,1, \ldots, N-1\right\}$, whose $n$-th sample is expressed by eq. (10) with $L=1$, i.e. as

$$
x_{r, n}=a \cos (2 \pi n F+\psi)+w_{r, n}
$$

or, equivalently, as (see eq. (3))

$$
x_{r, n}=C \exp (j 2 \pi n F)+C^{*} \exp (-j 2 \pi n F)+w_{r, n},
$$

where (see eq. (4))

$$
C \triangleq \frac{A}{2}=\frac{a}{2} \exp (j \psi)
$$

and $F$ are the complex amplitude and the normalised frequency, respectively, of the tone itself. It is well known that the ML estimates $F_{\mathrm{ML}}$ and $C_{\mathrm{ML}}$ of the parameters $F$ and $C$, respectively, represent the solution of the least square problem (e.g., see $[35$, eq. (22)])

$$
\left(F_{\mathrm{ML}}, C_{\mathrm{ML}}\right) \triangleq \arg \min _{\tilde{F}, \tilde{C}} \varepsilon(\tilde{F}, \tilde{C})
$$

where $\tilde{F}$ and $\tilde{C}$ represent trial values of $F$ and $C$, respectively,

$$
\varepsilon(\tilde{F}, \tilde{C}) \triangleq \frac{1}{N} \sum_{n=0}^{N-1} \varepsilon_{n}(\tilde{F}, \tilde{C})
$$

is the mean square error (MSE) evaluated over the whole observation interval,

$$
\varepsilon_{n}(\tilde{F}, \tilde{C}) \triangleq\left[x_{r, n}-s_{n}(\tilde{F}, \tilde{C})\right]^{2}
$$

is the square error between the noisy sample $x_{r, n}$ (28) and its useful component

$$
s_{n}(\tilde{F}, \tilde{C}) \triangleq \tilde{C} \exp (j 2 \pi n \tilde{F})+\tilde{C}^{*} \exp (-j 2 \pi n \tilde{F}),
$$

evaluated under the assumption that $F=\tilde{F}$ and $C=\tilde{C}$.

The cost function $\varepsilon(\tilde{F}, \tilde{C})$ (31) can be easily reformulated in a different way as follows. To begin, substituting the RHS of eq. (33) in that of eq. (32) produces, after some manipulation,

$$
\begin{aligned}
\varepsilon_{n}(\tilde{F}, \tilde{C}) & =x_{r, n}^{2}+2\left[\tilde{C}_{R}^{2}+\tilde{C}_{I}^{2}\right] \\
& -4 x_{r, n}\left[\tilde{C}_{R} \cos \left(\tilde{\phi}_{n}\right)-\tilde{C}_{I} \sin \left(\tilde{\phi}_{n}\right)\right] \\
& +2\left[\left(\tilde{C}_{R}^{2}-\tilde{C}_{I}^{2}\right) \cos \left(2 \tilde{\phi}_{n}\right)-2 \tilde{C}_{R} \tilde{C}_{I} \sin \left(2 \tilde{\phi}_{n}\right)\right]
\end{aligned}
$$

where $\tilde{C}_{R} \triangleq \Re\{\tilde{C}\}, \tilde{C}_{I} \triangleq \Im\{\tilde{C}\}$,

$$
\tilde{\phi}_{n} \triangleq 2 \pi n \tilde{F}
$$

and $\Re\{x\}$ ( $\{x\}$ ) denotes the real (imaginary) part of the complex variable $x$. Then, substituting the RHS of eq. (34) in that of eq. (31) yields

$$
\begin{aligned}
\varepsilon(\tilde{F}, \tilde{C}) & =\varepsilon_{x}+2\left[\left(\tilde{C}_{R}^{2}-\tilde{C}_{I}^{2}\right) g_{R}(\tilde{F})+2 \tilde{C}_{R} \tilde{C}_{I} g_{I}(\tilde{F})\right] \\
& +2\left[\tilde{C}_{R}^{2}+\tilde{C}_{I}^{2}\right]-4\left[\tilde{C}_{R} \bar{X}_{R}(\tilde{F})+\tilde{C}_{I} \bar{X}_{I}(\tilde{F})\right]
\end{aligned}
$$


where

$$
\varepsilon_{x} \triangleq \frac{1}{N} \sum_{n=0}^{N-1} x_{r, n}^{2}
$$

$\bar{X}_{R}(\tilde{F}) \triangleq \Re\{\bar{X}(\tilde{F})\}, \bar{X}_{I}(\tilde{F}) \triangleq \Im\{\bar{X}(\tilde{F})\}$,

$$
\begin{aligned}
\bar{X}(\tilde{F}) & \triangleq \frac{1}{N} \sum_{n=0}^{N-1} x_{r, n} \exp \left(-j \tilde{\phi}_{n}\right) \\
& =\frac{1}{N} \sum_{n=0}^{N-1} x_{r, n} \exp (-j 2 \pi n \tilde{F}),
\end{aligned}
$$

is, up to the scale factor $1 / N$, the Fourier transform of the sequence $\left\{x_{r, n}\right\}, g_{R}(\tilde{F}) \triangleq \Re\{g(\tilde{F})\}, g_{I}(\tilde{F}) \triangleq \Im\{g(\tilde{F})\}$ and

$$
g(\tilde{F}) \triangleq \frac{1}{N} \sum_{n=0}^{N-1} \exp \left(-j 2 \tilde{\phi}_{n}\right)=\frac{1}{N} \sum_{n=0}^{N-1} \exp (-j 4 \pi n \tilde{F}) .
$$

Based on eq. (36), it is not difficult to show that the optimization problem expressed by eq. (30) does not admit a closed form solution because of the nonlinear dependence of the function $\varepsilon(\tilde{F}, \tilde{C})$ on its variable $\tilde{F}$. However, an approximate solution to this problem can be derived by

a) Exploiting an iterative method, known as alternating minimization (AM) [63]. This allows us to transform the twodimensional (2D) optimization problem expressed by eq. (30) into a couple of interconnected one-dimensional (1D) problems, one involving the variable $\tilde{F}$ only (conditioned on the knowledge of $\tilde{C}$ ), the other one involving the variable $\tilde{C}$ only (conditioned on the knowledge of $\tilde{F}$ ).

b) Expressing the dependence of the function $\varepsilon(\tilde{F}, \tilde{C})$ on the variable $\tilde{F}$ through the couple $\left(F_{c}, \tilde{\delta}\right)$ such that

$$
\tilde{F}=F_{c}+\tilde{\delta} F_{\mathrm{DFT}},
$$

where $F_{c}$ is a given coarse estimate of $F, \tilde{\delta}$ is a real variable called residual,

$$
F_{\text {DFT }}=\frac{1}{N_{0}}
$$

is the normalized fundamental frequency associated with the $N_{0}$-th order discrete Fourier transform (DFT)

$$
\mathbf{X}_{0}=\left[X_{0,0}, X_{0,1}, \ldots, X_{0, N_{0}-1}\right]^{T}
$$

of the zero padded version

$$
\mathbf{x}_{0, \mathrm{ZP}}=\left[\mathbf{x}_{0}^{T} \mathbf{0}_{(M-1) N}^{T}\right]^{T}
$$

of the vector

$$
\mathbf{x}_{0} \triangleq\left[x_{r, 0}, x_{r, 1}, \ldots, x_{r, N-1}\right]^{T}
$$

collecting all the elements of the sequence $\left\{x_{r, n}\right\}, M$ is a positive integer (dubbed oversampling factor), $\mathbf{0}_{D}$ is a $D$-dimensional (column) null vector and $N_{0} \triangleq M \cdot N$.

c) Expressing the dependence of the function $\varepsilon(\tilde{F}, \tilde{C})$ (36) on the variable $\tilde{\delta}$ through its powers $\left\{\tilde{\delta}^{l} ; 0 \leq l \leq 3\right\}$; this result is achieved by approximating various trigonometric functions appearing in the expression of $\varepsilon(\tilde{F}, \tilde{C})$ with their Taylor expansions truncated to a proper order.

Let us show now how these principles can be put into practice. First of all, the exploitation of the above mentioned AM approach requires solving the following two sub-problems: P1) minimizing the cost function $\varepsilon(\tilde{F}, \tilde{C})(36)$ with respect to $\tilde{C}$, given $\tilde{F}=\hat{F} ; \mathbf{P 2}$ ) minimizing the same function with respect to $\tilde{F}$, given $\tilde{C}=\hat{C}$. Sub-problem P1 can be easily solved thanks to the polynomial dependence of the cost function $\varepsilon(\hat{F}, \tilde{C})$ on the variables $\tilde{C}_{R}$ and $\tilde{C}_{I}$. In fact, the minimum of the function $\varepsilon(\tilde{F}, \tilde{C})$ with respect to the last variables is the solution of the equations

$$
\tilde{C}_{R}-\bar{X}_{R}(\hat{F})+\tilde{C}_{R} g_{R}(\hat{F})+\tilde{C}_{I} g_{I}(\hat{F})=0
$$

and

$$
\tilde{C}_{I}-\bar{X}_{I}(\hat{F})-\tilde{C}_{I} g_{R}(\hat{F})+\tilde{C}_{R} g_{I}(\hat{F})=0,
$$

that result from computing the partial derivative of the RHS of eq. (36) with respect to $\tilde{C}_{R}$ and $\tilde{C}_{I}$, respectively. Solving the linear system of equations (45)-(46) in the unknowns $\tilde{C}_{R}$ and $\tilde{C}_{I}$ produces the optimal values

$$
\hat{C}_{R}=\frac{\bar{X}_{R}(\hat{F})\left[1-g_{R}(\hat{F})\right]-\bar{X}_{I}(\hat{F}) g_{I}(\hat{F})}{1-|g(\hat{F})|^{2}}
$$


and

$$
\hat{C}_{I}=\frac{-\bar{X}_{R}(\hat{F}) g_{I}(\hat{F})+\bar{X}_{I}(\hat{F})\left[1+g_{R}(\hat{F})\right]}{1-|g(\hat{F})|^{2}} .
$$

of $\tilde{C}_{R}$ and $\tilde{C}_{I}$, respectively. Putting together the last two formulas yields

$$
\hat{C}=\hat{C}_{R}+j \hat{C}_{I}=\frac{\bar{X}(\hat{F})-\bar{X}^{*}(\hat{F}) g(\hat{F})}{1-|g(\hat{F})|^{2}} .
$$

Therefore, given $\tilde{F}=\hat{F}$, the optimal value $\hat{C}$ of the variable $\tilde{C}$ can be computed exactly through the last equation; this requires the evaluation of $\bar{X}(\hat{F})$ and $g(\hat{F})$ (see eqs. (38) and (39), respectively). Note that, on the one hand, $g(\hat{F})$ can be easily evaluated through its exact expression

$$
g(\hat{F})=\frac{1}{N} \frac{\exp (-j 4 \pi N \hat{F})-1}{\exp (-j 4 \pi \hat{F})-1},
$$

which is easily derived from its definition (39). On the other hand, $\bar{X}(\hat{F})$ can be computed exactly through its expression (38) or, in an approximate fashion, through a computationally efficient procedure based on the fact that the vector

$$
\overline{\mathbf{X}}_{s} \triangleq M \mathbf{X}_{0}
$$

collects $N_{0}$ uniformly spaced samples of the function $\bar{X}(F)$ (38). In fact, the $k$-th element

$$
\bar{X}_{0, k} \triangleq \frac{1}{N_{0}} \sum_{n=0}^{N-1} x_{r, n} \exp \left(-j \frac{2 \pi n k}{N_{0}}\right),
$$

of the vector $\mathbf{X}_{0}$ (42) can be expressed as

$$
\bar{X}_{0, k}=\frac{N}{N_{0}} \bar{X}\left(F_{k}\right)=\frac{1}{M} \bar{X}\left(F_{k}\right)
$$

where

$$
F_{k} \triangleq k F_{\mathrm{DFT}}
$$

with $k=0,1, \ldots, N_{0}-1$. For this reason, an approximate evaluation of the quantity $\bar{X}(\hat{F})$ at a normalised frequency $\hat{F}$ different from any multiple of $F_{\mathrm{DFT}}(41)$ can be accomplished by interpolating the elements of the vector $\overline{\mathbf{X}}_{s}(51)$; the last vector, in turn, can be easily computed after evaluating the $N_{0}$-th fast Fourier transform (FFT) of $\mathbf{x}_{0, \mathrm{ZP}}$ (43), i.e. the vector $\mathbf{X}_{0}$ (42).

Let us take into consideration now sub-problem P2. Unluckily, this sub-problem, unlike the previous one, does not admit a closed form solution. For this reason, an approximate solution is developed below. Such a solution is based on representing the normalized frequency $F$ in the same form as $\tilde{F}$ (see eq. (40)), i.e. as

$$
F=F_{c}+\delta F_{\mathrm{DFT}}
$$

and on a novel method for estimating the real residual $\delta$, i.e. for accomplishing the fine estimation of $F$. This method is derived as follows. Representing the trial normalized frequency $\tilde{F}$ according to eq. (40) allows us to express the variable $\tilde{\phi}_{n}$ (35) as

$$
\tilde{\phi}_{n}=\hat{\theta}_{n}+n \tilde{\Delta}
$$

where

$$
\tilde{\Delta} \triangleq 2 \pi \tilde{\delta} F_{\mathrm{DFT}}
$$

is a new variable and

$$
\hat{\theta}_{n} \triangleq 2 \pi n F_{c}
$$

Then, substituting the RHS of eq. (56) in that of eq. (34) (with $\tilde{C}=\hat{C}$ ) yields

$$
\begin{aligned}
\varepsilon_{n}(\tilde{F}, \hat{C}) & =x_{r, n}^{2}+2\left[\hat{C}_{R}^{2}+\hat{C}_{I}^{2}\right] \\
& -4 x_{r, n}\left[\hat{C}_{R} \cos \left(n \tilde{\Delta}+\hat{\theta}_{n}\right)-\hat{C}_{I} \sin \left(n \tilde{\Delta}+\hat{\theta}_{n}\right)\right] \\
& +2\left[\left(\hat{C}_{R}^{2}-\hat{C}_{I}^{2}\right) \cos \left(2 n \tilde{\Delta}+2 \hat{\theta}_{n}\right)\right. \\
& \left.-2 \hat{C}_{R} \hat{C}_{I} \sin \left(2 n \tilde{\Delta}+2 \hat{\theta}_{n}\right)\right] .
\end{aligned}
$$


Based on the last equation and some trigonometric approximations (see Appendix A), the approximate expression

$$
\begin{aligned}
& \varepsilon_{\mathrm{SFE}}(\tilde{\Delta}, \hat{C}) \triangleq \\
& \varepsilon_{x}+2\left[\hat{C}_{R}^{2}+\hat{C}_{I}^{2}\right]-2 \hat{C}_{R}\left[2 X_{0, \rho}^{(R)}+2 \tilde{\Delta} X_{1, \rho}^{(I)}-\tilde{\Delta}^{2} X_{2, \rho}^{(R)}\right] \\
& -2 \hat{C}_{I}\left[2 X_{0, \rho}^{(I)}-2 \tilde{\Delta} X_{1, \rho}^{(R)}-\tilde{\Delta}^{2} X_{2, \rho}^{(I)}\right]+2\left(\hat{C}_{R}^{2}-\hat{C}_{I}^{2}\right) \\
& {\left[K_{0}^{(R)}(2 \rho)+2 \tilde{\Delta} K_{1}^{(I)}(2 \rho)-2 \tilde{\Delta}^{2} K_{2}^{(R)}(2 \rho)+\right.} \\
& \left.-\frac{4}{3} \tilde{\Delta}^{3} K_{3}^{(I)}(2 \rho)\right]-4 \hat{C}_{R} \hat{C}_{I}\left[-K_{0}^{(I)}(2 \rho)+2 \tilde{\Delta} K_{1}^{(R)}(2 \rho)+\right. \\
& \left.+2 \tilde{\Delta}^{2} K_{2}^{(I)}(2 \rho)-\frac{4}{3} \tilde{\Delta}^{3} K_{3}^{(R)}(2 \rho)\right]
\end{aligned}
$$

can be derived for the function $\varepsilon(\tilde{F}, \hat{C})(36)$; here,

$$
\begin{aligned}
& \rho \triangleq F_{c} / F_{\mathrm{DFT}} \\
& X_{k, \rho}^{(R)} \triangleq \Re\left\{X_{k, \rho}\right\}, X_{k, \rho}^{(I)} \triangleq \Im\left\{X_{k, \rho}\right\}, K_{p}^{(R)}(x) \triangleq \Re\left\{K_{p}(x)\right\}, K_{p}^{(I)}(x) \triangleq \Im\left\{K_{p}(x)\right\} \text { for any } k, p \text { and } x \geq 0, \\
& K_{p}(x) \triangleq \frac{1}{N} \sum_{n=0}^{N-1} g_{p}[n] \exp \left(-j \frac{2 \pi n x}{N_{0}}\right),
\end{aligned}
$$

$g_{p}[n] \triangleq n^{p}$ for $p=1,2$ and $3, g_{0}[n] \triangleq 1$

$$
\bar{X}_{k, \rho} \triangleq \frac{1}{N_{0}} \sum_{n=0}^{N-1} x_{k, n} \exp \left(-j \frac{2 \pi n \rho}{N_{0}}\right)
$$

for any $\rho$ and $k=1,2$, and

$$
x_{k, n} \triangleq n^{k} \cdot x_{r, n}
$$

with $n=0,1, \ldots, N-1$. It is important to point out that: a) if $\rho$ is an integer, the quantity $\bar{X}_{k, \rho}(63)$ (with $k=1$ and 2 ) represents the $\rho$-th element of the vector

$$
\mathbf{X}_{k}=\left[X_{k, 0}, X_{k, 1}, \ldots, X_{k, N_{0}-1}\right]^{T}
$$

generated by the $N_{0}$-th order DFT of the zero padded version

$$
\mathbf{x}_{k, \mathrm{ZP}}=\left[\mathbf{x}_{k}^{T} \mathbf{0}_{(M-1) N}^{T}\right]^{T}
$$

of the vector

$$
\mathbf{x}_{k} \triangleq\left[x_{k, 0}, x_{k, 1}, \ldots, x_{k, N-1}\right]^{T} ;
$$

b) if $\rho$ is not an integer, the quantity $\bar{X}_{k, \rho}$ can be evaluated exactly on the basis of eq. (63) or, in an approximate fashion, by interpolating a subset of adjacent elements of the vector $\mathbf{X}_{k}(65)$; c) the evaluation of the vectors $\left\{\mathbf{X}_{k} ; k=1,2\right\}$ requires two additional FFTs.

Since the function $\varepsilon_{\mathrm{SFE}}(\tilde{\Delta}, \hat{C})(60)$ is a polynomial of degree three in the variable $\tilde{\Delta}$, an estimate $\hat{\Delta}$ of $\Delta$ and, consequently, an estimate (see eq. (57))

$$
\hat{\delta}=\frac{\hat{\Delta}}{2 \pi F_{\mathrm{DFT}}}
$$

of $\delta$, can be obtained by computing the derivative of this function with respect to $\tilde{\Delta}$, setting it to zero and solving the resulting quadratic equation

$$
a(\rho) \tilde{\Delta}^{2}+b(\rho) \tilde{\Delta}+c(\rho)=0
$$

in the variable $\tilde{\Delta}$; here,

$$
\begin{gathered}
a(\rho) \triangleq 2 \Im\left\{\left(\hat{C}^{*}\right)^{2} K_{3}(2 \rho)\right\} \\
b(\rho) \triangleq-\Re\left\{\hat{C}^{*} \bar{X}_{2, \rho}\right\}+2 \Re\left\{\left(\hat{C}^{*}\right)^{2} K_{2}(2 \rho)\right\}
\end{gathered}
$$

and

$$
c(\rho) \triangleq \Im\left\{\hat{C}^{*} \bar{X}_{1, \rho}\right\}-\Im\left\{\left(\hat{C}^{*}\right)^{2} K_{1}(2 \rho)\right\} .
$$


Note that only one of the two solutions of eq. (69), namely

$$
\hat{\Delta}=-\frac{b(\rho)+\sqrt{(b(\rho))^{2}-4 a(\rho) c(\rho)}}{2 a(\rho)}
$$

has to be employed. A simpler estimate of $\Delta$ is obtained neglecting the contribution of the first term in the left-hand side of eq. (69), i.e. setting $a(\rho)=0$. This leads to a first-degree equation, whose solution is

$$
\hat{\Delta}=-c(\rho) / b(\rho) \text {. }
$$

Given an estimate $\hat{\Delta}$ of $\Delta$ (and, consequently, and estimate $\hat{\delta}$ of $\delta$; see eq. (68)), the fine estimate

$$
\hat{F}=F_{c}+\hat{\delta} F_{\text {DFT }}=F_{c}+\frac{\hat{\Delta}}{2 \pi}
$$

of $F$ can be evaluated on the basis of eq. (55).

The mathematical results derived above allow us to derive a novel estimation algorithm, called single frequency estimator (SFE), for iteratively estimating the normalised frequency $F$ and the complex amplitude $C$. This algorithm is initialised by

1) Evaluating: a) the vector $\mathbf{X}_{0}(42)$; b) the initial coarse estimate $\hat{F}_{c}^{(0)}$ of $F$ as

$$
\hat{F}_{c}^{(0)}=\hat{\alpha} F_{\mathrm{DFT}}
$$

where the integer $\hat{\alpha}$ is computed by means of the well known periodogram method (e.g., see [35, Sec. IV] or [40, Sec. I]), i.e. as

$$
\hat{\alpha}=\arg \max _{\tilde{\alpha} \in\left\{0,1, \ldots, N_{0} / 2\right\}}\left|\bar{X}_{0, \tilde{\alpha}}\right|
$$

c) the quantity (see eq. (61))

$$
\hat{\rho}^{(0)} \triangleq \frac{\hat{F}_{c}^{(0)}}{F_{\mathrm{DFT}}}=\hat{\alpha}
$$

d) the initial estimate $\hat{C}^{(0)}$ of $C$ on the basis of eq. (49) with $\hat{F}=\hat{F}_{c}^{(0)}$; e) the spectral coefficients $\bar{X}_{1, \hat{\alpha}}$ and $\bar{X}_{2, \hat{\alpha}}$ on the basis of eq. (63); f) the coefficients $\left\{K_{p}(2 \hat{\alpha}) ; p=1,2\right\}\left(\left\{K_{p}(2 \hat{\alpha}) ; p=1,2,3\right\}\right)$ according to eq. (62), the coefficients $\{a(\hat{\alpha})$, $b(\hat{\alpha}), c(\hat{\alpha})\}\left(\{b(\hat{\alpha}), c(\hat{\alpha})\}\right.$ according to eqs. (70)-(72) and the first estimate $\hat{\Delta}^{(0)}$ of $\Delta$ on the basis of eq. (73) (eq. (74)); g) the first fine estimate $\hat{F}^{(0)}$ of $F$ on the basis of eqs. (55) and (68), i.e. as

$$
\hat{F}^{(0)}=\hat{F}_{c}^{(0)}+\frac{\hat{\Delta}^{(0)}}{2 \pi}
$$

2) Setting its iteration index $i$ to 1 .

Then, an iterative procedure is started. The $i$-th iteration is fed by the estimates $\hat{F}^{(i-1)}$ and $\hat{C}^{(i-1)}$ of $F$ and $C$, respectively, and produces the new estimates $\hat{F}^{(i)}$ and $\hat{C}^{(i)}$ of the same quantities (with $i=1,2, \ldots, N_{\mathrm{SFE}}$, where $N_{\mathrm{SFE}}$ is the overall number of iterations); the procedure employed for the evaluation of $\hat{F}^{(i)}$ and $\hat{C}^{(i)}$ consists of the two steps described below (the $p$-th step is denoted SFE-S $p$ ).

SFE-S1 - The new estimate $\hat{\Delta}^{(i)}$ of $\Delta$ is computed through eq. (73) (eq. (74)); in the evaluation of the coefficients $\{a(\rho)$, $b(\rho), c(\rho)\}(\{b(\rho), c(\rho)\})$ appearing in the RHS of these equations, $\hat{C}=\hat{C}^{(i-1)}$ and

$$
\rho=\hat{\rho}^{(i-1)} \triangleq \hat{F}^{(i-1)} / F_{\mathrm{DFT}}
$$

are assumed. Then,

$$
\hat{F}^{(i)}=\hat{F}^{(i-1)}+\frac{\hat{\Delta}^{(i)}}{2 \pi}
$$

is evaluated.

SFE-S2 - The new estimate $\hat{C}^{(i)}$ of $\hat{C}$ is evaluated through eq. (49); $\hat{F}=\hat{F}^{(i)}$ is assumed in this case. Moreover, the index $i$ is incremented by one before starting the next iteration.

At the end of the last (i.e., of the $N_{\mathrm{SFE}}$-th) iteration, the fine estimates $\hat{F}=\hat{F}^{\left(N_{\mathrm{SFE}}\right)}$ and $\hat{C}=\hat{C}^{\left(N_{\mathrm{SFE}}\right)}$ of $F$ and $C$, respectively, become available.

The SFE is summarized in Algorithm 1. It is important to point out that:

a) The estimate $\hat{\delta}^{(i)}$ of the residual $\delta$ computed by the SFE in its $i$-th iteration is expected to become smaller as $i$ increases, since $\hat{F}^{(i)}$ should progressively approach $F$ if our algorithm converges.

b) The coefficients $\left\{K_{p}(2 \rho) ; p=1,2,3\right\}$ can be computed exactly on the basis of eq. (62). However, since the definition (62) can be put in the equivalent form

$$
K_{p}(x) \triangleq \frac{1}{N} \sum_{n=0}^{N-1} n^{p}(q(x))^{n}
$$


where

$$
q(x) \triangleq \exp \left(-j \frac{2 \pi x}{N_{0}}\right)
$$

the identities

$$
\begin{gathered}
(q-1)^{2} \sum_{n=0}^{N-1} n q^{n}=(N-1) q^{N+1}-N q^{N}+q, \\
(q-1)^{3} \sum_{n=0}^{N-1} n^{2} q^{n}=(N-1)^{2} q^{N+2}+\left(-2 N^{2}+2 N+1\right) q^{N+1} \\
+N^{2} q^{N}-q^{2}-q
\end{gathered}
$$

and

$$
\begin{aligned}
(q-1)^{4} & \sum_{n=0}^{N-1} n^{3} q^{n}=q+4 q^{2}+q^{3}-N^{3} q^{N} \\
& +\left(3 N^{3}-3 N^{2}-3 N-1\right) q^{N+1} \\
& +\left(-3 N^{3}+6 N^{2}-4\right) q^{N+2}+(N-1)^{3} q^{N+3}
\end{aligned}
$$

holding for any $q \in \mathbb{C}$, can be exploited for the efficient computation of $K_{p}(2 \rho)$ for any $p$ and $\rho$.

c) The quantities $\left\{\bar{X}_{k, \hat{\rho}^{(i-1)}} ; k=1,2\right\}$ required in the first step of the $i$-th iteration can be computed exactly on the basis of eq. (63). However, they can be also evaluated, in an approximate fashion, by interpolating $I$ adjacent elements of the $N_{0}$-dimensional vectors $\mathbf{X}_{k}(65)$, where $I$ denotes the selected interpolation order.

d) The estimate $\hat{\Delta}^{(i)}$ evaluated according to eq. (74) is expected to be less accurate than that computed on the basis of eq. (73). However, our numerical results have evidenced that both solutions achieve similar accuracy. Despite, the use of eq. (73) is assumed in Algorithm 1 for generality.

e) The SFE can be employed even if the single tone appearing in the RHS of eq. (27) is replaced by the superposition of $L$ distinct tones (see eq. (3)). In this case, the strongest (i.e., the dominant) tone is detected through the periodogram method (see eq. (77)) and the parameters of this tone are estimated in the presence of both Gaussian noise and the interference due to the remaining tones. Therefore, the estimation accuracy of the SFE is affected by both the amplitudes and the frequencies of the other $(L-1)$ tones.

f) A stopping criterion, based on the trend of the sequence $\left\{\hat{\Delta}^{(i)} ; i=1,2, \ldots\right\}$, can be easily formulated for the SFE. For instance, the execution of its two steps can be stopped if, at the end of the $i$-th iteration, the condition

$$
\left|\hat{\Delta}^{(i)}\right|<\varepsilon_{\Delta}
$$

is satisfied; here, $\varepsilon_{\Delta}$ represents a proper threshold.

All the results developed above refer to the real sequence $\left\{x_{r, n}\right\}$, whose $n$-th element is expressed by eq. (27). However, a similar estimation method (dubbed complex SFE, CSFE) can be developed for the complex counterpart, i.e. for a complex sequence $\left\{x_{c, n}\right\}$ such that (see eq. (15) with $L=1$ )

$$
x_{c, n}=A \exp (j 2 \pi n F)+w_{c, n},
$$

with $n=0,1, \ldots, N-1$. In this case, the ML estimation of the parameters $F$ and $A$ can be formulated in a similar way as eq. (30), the only differences being represented by the fact that: a) the parameter $C$ is replaced by $A$; b) the term $\varepsilon_{n}(\tilde{F}, \tilde{C})$ appearing in the RHS of eq. (31) is replaced by

$$
\varepsilon_{n}(\tilde{F}, \tilde{A}) \triangleq\left|x_{c, n}-s_{n}(\tilde{F}, \tilde{A})\right|^{2},
$$

where

$$
s_{n}(\tilde{F}, \tilde{A}) \triangleq \tilde{A} \exp (j 2 \pi n \tilde{F})
$$

represents the useful component of $x_{c, n}$ (89) under the assumption that $F=\tilde{F}$ and $A=\tilde{A}$. Substituting the RHS of the last equation in that of eq. (90) and, then, the resulting expression in the RHS of the MSE (see eq. (1))

$$
\varepsilon(\tilde{F}, \tilde{A}) \triangleq \frac{1}{N} \sum_{n=0}^{N-1} \varepsilon_{n}(\tilde{F}, \tilde{A})
$$

yields, after some manipulation,

$$
\varepsilon(\tilde{F}, \tilde{A})=\varepsilon_{x}+\tilde{A}_{R}^{2}+\tilde{A}_{I}^{2}-2 \tilde{A}_{R} \bar{X}_{R}(\tilde{F})-2 \tilde{A}_{I} \bar{X}_{I}(\tilde{F}),
$$


where

$$
\varepsilon_{x} \triangleq \frac{1}{N} \sum_{n=0}^{N-1}\left|x_{c, n}\right|^{2} .
$$

The procedure adopted to minimise the last function with respect to $\tilde{F}$ and $\tilde{A}$ is similar to that illustrated above for the SFE. For this reason, in the following we limit to show essential mathematical results only. First all, it is easy to show that, given $\tilde{F}=\hat{F}$, the function $\varepsilon(\hat{F}, \tilde{A})(93)$ is minimised with respect to $\tilde{A}$ selecting ${ }^{2}$

$$
\tilde{A}=\hat{A}=\bar{X}(\hat{F}),
$$

where $\bar{X}(\hat{F})$ can be computed exactly through its expression (see eq. (38)) replacing the real sequence $\left\{x_{r, n}\right\}$ with the complex sequence $\left.\left\{x_{c, n}\right\}\right)$ or, in an approximate fashion, through an interpolation technique following the same mathematical approach as that illustrated for the SFE. Note also that the mathematical result expressed by eq. (95) is exact. On the contrary, given $\tilde{A}=\hat{A}$, a closed form expression for the value of $\tilde{F}$ minimising the function $\varepsilon(\tilde{F}, \hat{A})$ cannot be derived because of the nonlinear dependence of this function on $\tilde{F}$. However, following the same mathematical approach as that illustrated for the SFE, the approximate expression

$$
\begin{aligned}
\varepsilon_{\mathrm{CSFE}}(\tilde{\Delta}, \hat{A}) & \triangleq \varepsilon_{x}+\hat{A}_{R}^{2}+\hat{A}_{I}^{2} \\
& +\frac{1}{3} \tilde{\Delta}^{3}\left(\hat{A}_{R} \bar{X}_{3, \rho}^{(I)}-\hat{A}_{I} \bar{X}_{3, \rho}^{(R)}\right) \\
& +\tilde{\Delta}^{2}\left(\hat{A}_{R} \bar{X}_{2, \rho}^{(R)}+\hat{A}_{I} \bar{X}_{2, \rho}^{(I)}\right) \\
& +2 \tilde{\Delta}\left(\hat{A}_{I} \bar{X}_{1, \rho}^{(R)}-\hat{A}_{R} \bar{X}_{1, \rho}^{(I)}\right) \\
& -2\left(\hat{A}_{R} \bar{X}_{0, \rho}^{(R)}+\hat{A}_{I} \bar{X}_{0, \rho}^{(I)}\right)
\end{aligned}
$$

can be obtained for the cost function $\varepsilon(\tilde{F}, \tilde{A})$ (93) (see Appendix A for further details); the quantities appearing in the last equation have the same meaning as the ones defined for eq. (60). It worth stressing that:

a) The considerations expressed about the evaluation of the quantities $\left\{\bar{X}_{k, \rho}\right\}$ in our derivation of the SFE apply to the CFSE too. However, in this case, three additional spectral coefficients, namely $\left\{\bar{X}_{k, \rho} ; k=1,2,3\right\}$, need to be computed.

b) The initial estimate $\hat{\rho}^{(0)}=\hat{\alpha}$ (see eq. (78)) of $\rho$ is evaluated in a similar way as the SFE (see eq. (77)), i.e., as

$$
\hat{\alpha}=\arg \max _{\tilde{\alpha} \in\left\{0,1, \ldots, N_{0}-1\right\}}\left|\bar{X}_{0, \tilde{\alpha}}\right| \text {. }
$$

The minimization of the function $\varepsilon_{\mathrm{CSFE}}(\tilde{F}, \tilde{A})(96)$ with respect to $\tilde{\Delta}$ is achieved by taking its partial derivative with respect to this variable and setting it to zero; this results again in the quadratic equation (69), whose coefficients are

$$
\begin{gathered}
a(\rho) \triangleq \frac{1}{2} \Im\left\{\hat{A}^{*} \bar{X}_{3, \rho}\right\}, \\
b(\rho) \triangleq \Re\left\{\hat{A}^{*} \bar{X}_{2, \rho}\right\}
\end{gathered}
$$

and

$$
c(\rho) \triangleq-\Im\left\{\hat{A}^{*} \bar{X}_{1, \rho}\right\} .
$$

Following the approach adopted in the development of the SFE and exploiting the mathematical results expressed by eq. (95) and by eqs. (98)-(100) allow us to easily derive the CSFE; this algorithm is summarized in Algorithm 2.

Finally, it is important to point out the estimates generated by the SFE and the CSFE algorithms are unbiased, provided the number of iterations they accomplish is large enough; a proof of this statement is provided in Appendix D for the CSFE.

\section{B. Estimation of multiple tones}

Let us analyse now in detail how the techniques derived in the previous paragraph can be exploited to estimate the multiple tones that form the useful component of the real input sequence $\left\{x_{r, n}\right\}$ (the complex sequence $\left\{x_{c, n}\right\}$ ), when its $n$-th sample is expressed by eq. (2) (eq. (1)) with $L>1$. The two recursive methods we develop to achieve this target are based on the following basic principles:

1) Tones are sequentially detected and estimated.

2) The detection of a new tone and the estimation of its parameters are based on the procedure developed for the SFE and the CFSE in the previous paragraph; in addition, a cancellation algorithm is incorporated in both these methods to

${ }^{2}$ This is a well known result (e.g., see [35, Sec. IV]). 

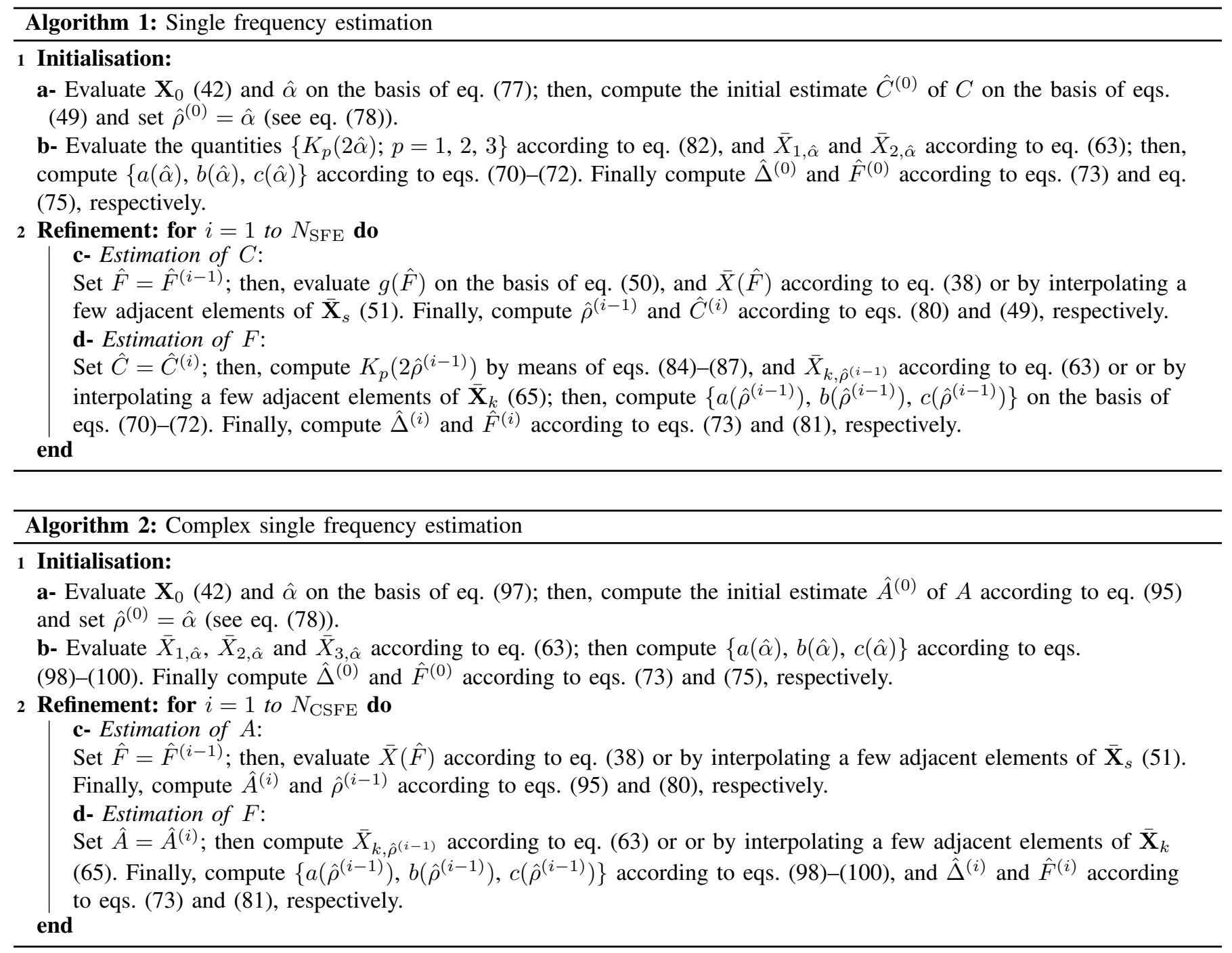

remove the contribution of previously detected tones from all the spectral information (namely, the spectrum $\bar{X}(F)(38)$, the vector $\mathbf{X}_{0}$ (42) and the coefficients $\left\{\bar{X}_{k, \rho}\right\}(63)$ ), that are processed to detect and estimate the new tone.

3) After detecting a new tone and estimating its parameters, a re-estimation technique is executed to improve the accuracy of both this tone and the previously estimated tones; the proposed technique is inspired by the related methods described in refs. [11], [21] and [22].

4) A proper criterion is adopted to stop recursions. This allows to estimate the (unknown) number of targets, that is the value of the parameter $L$.

The first recursive method relying on these principles is called single frequency estimation and cancellation (SFEC) or complex single frequency estimation and cancellation (CSFEC), depending on the fact that its input sequence is real or complex, respectively. In the following, for space limitations, we focus on the CSFEC method only; however, readers should keep in mind that the differences between this method and the SFEC are similar to those illustrated in our description of the single tone estimation algorithms on which they are based.

The CSFEC algorithm is initialised by:

1) Executing the CSFE, fed by the complex sequence $\left\{x_{c, n}\right\}$, to generate, through $N_{\mathrm{CSFE}}$ iterations, the initial estimates $\hat{F}_{0}[0]$ and $\hat{A}_{0}[0]$ of the normalized frequency and the complex amplitude, respectively, of the first detected tone.

2) Setting the recursion index $r$ to 1 .

Then, a recursive procedure is started. The $r$-th recursion is fed by the vectors

$$
\hat{\mathbf{F}}[r-1]=\left[\hat{F}_{0}[r-1], \hat{F}_{1}[r-1], \ldots, \hat{F}_{r-1}[r-1]\right]^{T},
$$

and

$$
\hat{\mathbf{A}}[r-1]=\left[\hat{A}_{0}[r-1], \hat{A}_{1}[r-1], \ldots, \hat{A}_{r-1}[r-1]\right]^{T},
$$


collecting the frequencies and the associated complex amplitudes characterizing the $r$ tones detected and estimated in the previous $(r-1)$ recursions, and generates the new vectors

$$
\hat{\mathbf{F}}[r]=\left[\hat{F}_{0}[r], \hat{F}_{1}[r], \ldots, \hat{F}_{r}[r]\right]^{T}
$$

and

$$
\hat{\mathbf{A}}[r]=\left[\hat{A}_{0}[r], \hat{A}_{1}[r], \ldots, \hat{A}_{r}[r]\right]^{T}
$$

after: a) estimating the frequency $\hat{F}_{r}[r]$ and the associated complex amplitude $\hat{A}_{r}[r]$ of a new (i.e., of the $r$-th) tone (if any); b) refining the estimates of the $r$ tones available at the beginning of the considered recursion. The procedure employed for accomplishing all this consists of the three steps described below (the $p$-th step is denoted CSFEC-S $p$ ).

CSFEC-S1 (CFSE initialisation with cancellation) - In this step, the following quantities are evaluated (see the initialisation part of Algorithm 2):

a) The residual spectrum

$$
\begin{aligned}
\mathbf{X}_{0}[r] & =\left[X_{0,0}[r], X_{0,1}[r], \ldots, X_{0, N_{0}-1}[r]\right]^{T} \\
& \triangleq \mathbf{X}_{0}-\mathbf{C}_{0}(\hat{\mathbf{A}}[r-1], \hat{\mathbf{F}}[r-1], r),
\end{aligned}
$$

where $\mathbf{X}_{0}$ is the $N_{0}$-th order DFT of the zero padded version $\mathbf{x}_{0, Z P}$ of the vector $\mathbf{x}_{0}$ collecting all the elements of the sequence $\left\{x_{c, n}\right\}$ (see eqs. (43)-(44)) and the $N_{0}$-dimensional vector

$$
\mathbf{C}_{0}(\hat{\mathbf{A}}[r-1], \hat{\mathbf{F}}[r-1], r) \triangleq \sum_{l=0}^{r-1} \overline{\mathbf{C}}_{0}\left(\hat{A}_{l}[r-1], \hat{F}_{l}[r-1]\right)
$$

represents the contribution given by all the estimated tones to $\mathbf{X}_{0}$ (in particular, $\overline{\mathbf{C}}_{0}\left(\hat{A}_{l}[r-1], \hat{F}_{l}[r-1]\right)$ is the contribution provided by $l$-th tone to the vector $\mathbf{X}_{0}$ (the expression of this vector can be found in Appendix B). If the overall energy

$$
\mathcal{E}_{0}[r] \triangleq\left|\mathbf{X}_{0}[r]\right|^{2}
$$

satisfies the inequality

$$
\mathcal{E}_{0}[r]<\mathcal{T}_{\mathrm{CSFEC}},
$$

where $\mathcal{T}_{C S F E C}$ is a proper threshold, the algorithm stops and the estimate $\hat{L}=r$ of $L$ is generated.

b) The integer (see eq. (97))

$$
\hat{\alpha}[r]=\arg \max _{\tilde{\alpha} \in\left\{0,1, \ldots, N_{0}-1\right\}}\left|X_{0, \tilde{\alpha}}[r]\right|,
$$

that represents the index of the element of $\mathbf{X}_{0}[r]$ (105) having the largest absolute value.

c) The preliminary estimate (see eq. (95))

$$
\bar{A}_{r}[r]=\bar{X}\left(\hat{F}_{c, r}[r]\right)-\bar{X}_{\mathrm{lk}, 0}\left(\hat{\mathbf{A}}[r-1], \hat{\mathbf{F}}[r-1], \hat{F}_{c, r}[r]\right)
$$

of the complex amplitude of the new tone; here,

$$
\hat{F}_{c, r}[r]=\hat{\alpha}[r] F_{\mathrm{DFT}}
$$

and

$$
\begin{aligned}
& \bar{X}_{\mathrm{lk}, 0}\left(\hat{\mathbf{A}}[r-1], \hat{\mathbf{F}}[r-1], \hat{F}_{c, r}[r]\right) \triangleq \\
& \sum_{l=0}^{r-1} \bar{X}_{0}\left(\hat{A}_{l}[r-1], \hat{F}_{l}[r-1], \hat{F}_{c, r}[r]\right)
\end{aligned}
$$

represent the coarse estimate of the frequency of the new tone (see eq. (76)) and the contribution given to $\bar{X}(F)$ by all the estimated tones (i.e., the leakage) at the frequency $F=\hat{F}_{c, r}[r]$ (in particular, $\bar{X}_{0}\left(\hat{A}_{l}[r-1], \hat{F}_{l}[r-1], \hat{F}_{c, r}[r]\right.$ ) is the leakage due to the $l$-th tone; the expression of the function $\bar{X}_{\mathrm{lk}, k}(\hat{A}, \hat{F}, \bar{F})$ is provided in Appendix B).

d) The spectral coefficient (see eq. (97))

$$
\bar{X}_{k, \rho[r]}[r]=\bar{X}_{k, \rho[r]}-\hat{X}_{\mathrm{lk}, k}\left(\hat{\mathbf{A}}[r-1], \hat{\mathbf{F}}[r-1], \hat{F}_{c, r}[r]\right)
$$

for $k=1,2$ and 3 ; here, we have that (see eq. (78))

$$
\rho[r]=\hat{F}_{c, r}[r] / F_{\mathrm{DFT}}=\hat{\alpha}[r]
$$


and

$$
\begin{aligned}
& \hat{X}_{\mathrm{lk}, k}\left(\hat{\mathbf{A}}[r-1], \hat{\mathbf{F}}[r-1], \hat{F}_{c, r}[r]\right) \triangleq \\
& \sum_{l=0}^{r-1} \bar{X}_{\mathrm{lk}, k}\left(\hat{A}_{l}[r-1], \hat{F}_{l}[r-1], \hat{F}_{c, r}[r]\right)
\end{aligned}
$$

is the contribution given to $\bar{X}_{k, \rho[r]}[r]$ by all the estimated tones (i.e., the leakage) at the frequency $\hat{F}_{c, r}[r]$ (in particular, $\bar{X}_{\mathrm{lk}, k}\left(\hat{A}_{l}[r-1], \hat{F}_{l}[r-1], \hat{F}_{c, r}[r]\right)$ represents the leakage due to the $l$-th estimated tone).

e) The coefficients $a(\hat{\alpha}[r]), b(\hat{\alpha}[r])$ and $c(\hat{\alpha}[r])$, the residual $\hat{\Delta}^{(0)}[r]$ and the normalized frequency

$$
\hat{F}_{r}^{(0)}=\hat{F}_{c, r}[r]+\frac{\hat{\Delta}^{(0)}[r]}{2 \pi}
$$

on the basis of eqs. (98)-(100), eq. (73) (or eq. (74)), and eqs. (76) and (79). Note that $\hat{F}_{r}^{(0)}$ represents the initial fine estimate of the normalized frequency of the new tone.

The evaluation of the frequency $\hat{F}_{r}^{(0)}(116)$ concludes the initialization of the modified CSFE executed for the detection and the estimation of the new tone.

CSFEC-S2 (CSFE refinement with cancellation) - After carrying out the first step, $N_{\mathrm{CSFE}}$ iterations ${ }^{3}$ are executed to refine the estimate of the parameters of the new tone. The processing accomplished in this step follows closely that described in the refinement part of Algorithm 2. For this reason, in each iteration, a new estimate of the complex amplitude and of the residual of frequency of the $r$-th tone are computed. This requires re-using eqs. (110)-(112) and (113), respectively, in order to remove the leakage in the spectrum $\bar{X}(F)$ and in the coefficients $\bar{X}_{k, \rho}$ (see steps $\mathbf{c}$ and $\mathbf{d}$, respectively, of Algorithm 2). At the end of the last iteration, the frequency $\hat{F}_{\mathrm{CSFE}, r}[r]$ and the associated complex amplitude $\hat{A}_{\mathrm{CSFE}, r}[r]$ of the new tone are available; these represent $\hat{F}_{r}[r]$ and $\hat{A}_{r}[r]$, respectively, if the following re-estimation step is not accomplished.

CSFEC-S3 (re-estimation) - This step is fed by the $(r+1)$ normalized frequencies $\left\{\hat{F}_{0}[r-1], \hat{F}_{1}[r-1], \ldots, \hat{F}_{r-1}[r-1]\right.$, $\left.\hat{F}_{\mathrm{CSFE}, r}[r]\right\}$ and the associated complex amplitudes $\left\{\hat{A}_{0}[r-1], \hat{A}_{1}[r-1], \ldots, \hat{A}_{r-1}[r-1], \hat{A}_{\mathrm{CSFE}, r}[r]\right\}$. It consists in repeating the previous step for each of the detected tones, starting from the first tone and ending with $(r+1)$-th one. This means that, when re-estimating the $l$-th tone, the leakage due to the tones whose index belong to set $\{0,1, . ., l-1, l+1, \ldots, r\}$ has to be removed by exploiting equations similar to (110) and (113)-(115), with $l=0,1, \ldots, r$. This allows us to progressively refine the amplitude and the frequency of each tone, so generating the final frequencies $\left\{\hat{F}_{0}[r], \hat{F}_{1}[r], \ldots, \hat{F}_{r}[r]\right\}$ and their complex amplitudes $\left\{\hat{A}_{0}[r], \hat{A}_{1}[r], \ldots, \hat{A}_{r}[r]\right\}$. Note that, in principle, the re-estimation can be accomplished multiple (say, $N_{\text {RES }}$ ) times.

Our simulation results have evidenced that, unluckily, the estimates generated by the SFEC and CSFEC algorithms are biased. However, this bias can be removed by running an additional (i.e., a fourth) step after that these algorithms have been executed. In this final step, the estimation algorithm developed by $Y e$ and Aboutanios in ref. [49], [50] is carried out after initializing it with the estimates $\left\{\hat{F}_{0}[\hat{L}], \hat{F}_{1}[\hat{L}], \ldots, \hat{F}_{\hat{L}-1}[\hat{L}]\right\}$ and their complex amplitudes $\left\{\hat{A}_{0}[\hat{L}], \hat{A}_{1}[\hat{L}], \ldots, \hat{A}_{\hat{L}-1}[\hat{L}]\right\}\left(\left\{\hat{C}_{0}[\hat{L}], \hat{C}_{1}[\hat{L}]\right.\right.$, $\left.\left.\ldots, \hat{C}_{\hat{L}-1}[\hat{L}]\right\}\right)$ generated by the CSFEC (CSFE). The hybrid techniques resulting from interconnecting the SFEC and CSFEC algorithms with the above mentioned algorithm are dubbed hybrid SFEC (HSFEC) and hybrid CSFEC (HCSFEC).

It is worth pointing out that:

a) The oversampling factor $M$ adopted in the computation of the vectors $\left\{\mathbf{X}_{k}^{(l)}\right\}$ and the stopping criterion employed by the SFE (or CSFE) need to be carefully adjusted in order to achieve a good accuracy in the estimation of the parameters of each new tone.

b) Poor estimation of the normalised frequency $F_{l}$ and/or the complex amplitude $A_{l}\left(C_{l}\right)$ may lead to significant error accumulation if CSFEC-S3 (the corresponding step in the SFEC) is removed; readers should always keep in mind that a fundamental role in accurate cancellation is played by the accuracy of the estimated frequency residual.

c) The threshold $\mathcal{T}_{\text {CSFEC }}$ needs to be properly adjusted in order to ensure that the probability that $\hat{L}$ equals to $L$ is close to unity. On the one hand, a large value of $\mathcal{T}_{\mathrm{CSFEC}}$ may lead to miss weaker tones; on the other hand, a small value of this parameter may lead to the identification of nonexistent tones and, consequently, of false targets in a radar system.

\section{Estimation of single and multiple delays}

All the estimation techniques developed in the previous two paragraphs refer to the real (complex) sequence $\left\{x_{r, n}\right\}\left(\left\{x_{c, n}\right\}\right)$, whose $n$-th element is expressed by eq. (10) (eq. (15)). As shown in Section II, these models are suitable to represent the sampled downconverted signal made available by the receiver of a colocated FMCW MIMO radar. However, similar estimation methods can be also developed for a colocated SFCW MIMO radar system, since the complex signal model (18) illustrated for this system is very similar to the model (15) referring to its complex FMCW counterpart, the only differences being represented by the fact that: a) the physical meaning of the parameter $F_{l}^{(v)}$, since this represents a normalized delay in eq. (18) and a

${ }^{3}$ The potential dependence of the parameter $N_{\mathrm{CSFE}}$ on the recursion index $r$ is ignored here for simplicity. 
normalized frequency in eq. (15); b) the sign of the argument of all the complex exponentials appearing in the RHS of eq. (18), since this is the opposite of that of the corresponding functions contained in the RHS of eq. (15). The implications of this sign reversal can be summarised as follows. In the derivation of the single tone estimator, the function $\bar{X}(\tilde{F})(38)$ is replaced by

$$
\bar{X}(\tilde{F}) \triangleq \frac{1}{N} \sum_{n=0}^{N-1} x_{c, n} \exp (j 2 \pi n \tilde{F}),
$$

where $x_{c, n}$ is the $n$-th sample of the received signal sequence (see eq. (18)). Consequently, the vectors $\mathbf{X}_{0}$ (42) and $\mathbf{X}_{k}(65)$ are generated by taking the $N_{0}$ order inverse DFT (IDFT) of the zero-padded vectors $\mathbf{x}_{0, \mathrm{ZP}}$ (43) and $\mathbf{x}_{k, \mathrm{ZP}}(66)$, respectively; note that the $n$-th element of the $N_{0}$-th dimensional vector $\mathbf{x}_{0, \mathrm{ZP}}\left(\mathbf{x}_{k, \mathrm{ZP}}\right)$ is equal to $x_{c, n}\left(n^{k} \cdot x_{c, n}\right.$; see eq. (64)) for $n=0$, $1, \ldots, N-1$ and is equal to zero for $n \geq N$. An algorithm for estimating a single delay can be easily developed by following the same line of reasoning as the CSFE; this is called complex single delay estimator (CSDE). More specifically, the CSDE computes an estimate $\hat{\delta}$ of the delay residual $\delta$ by solving eq. (69) in the variable $\tilde{\Delta}$ (57); in this case, the coefficients $a(\rho)$ and $c(\rho)$ are still expressed by eqs. (98) and (100), respectively, whereas

$$
b(\rho) \triangleq-\Re\left\{\hat{A}^{*} \bar{X}_{2, \rho}\right\},
$$

where $\hat{A}$ is the estimate of the complex gain $A$ computed on the basis of eq. (95). Moreover, in the evaluation of the coefficients $\{a(\rho), b(\rho), c(\rho)\}$ of eq. (69), the quantity $\bar{X}_{k, \hat{\rho}^{(i-1)}}$ (see eq. (63)) is replaced by

$$
\bar{X}_{k, \hat{\rho}^{(i-1)}} \triangleq \frac{1}{N} \sum_{n=0}^{N-1} x_{k, n} \exp \left(j \frac{2 \pi n \hat{\rho}^{(i-1)}}{N_{0}}\right),
$$

where $\hat{\rho}^{(i-1)}$ is still defined by eq. (80). These considerations must be kept into account in the development of the counterpart of the CSFEC algorithm; this is called complex single delay estimation and cancellation (CSDEC). Also in this case, the estimation algorithm developed by $Y e$ and Aboutanios in ref. [49], [50] is interconnected to our CSDEC algorithm; the resulting hybrid version is dubbed hybrid CSDEC (HCSDEC).

\section{Comparison with other estimation methods}

The SFEC and CSFEC techniques developed in the Paragraphs III-A-III-B are conceptually related to the multiple tone estimators developed by Gough [21], Li and Stoica [22], Macleod [11], Ye and Aboutanios [49], [50], Serbes and Qaraqe [51], and Serbes [69] (these algorithms are denoted CLEAN, RELAX, Alg-M, Alg-YA, CFH and Alg-S, respectively, in the following). In fact, all these algorithms are recursive and rely on a serial cancellation procedure since, within each recursion, they detect a single tone, estimate its parameters and subtract its contribution from the residual signal emerging from the previous iteration. Despite their similar structure, they exhibit various differences, that concern the three specific issues listed below.

Single frequency estimator -The main difference is represented by the algorithm they employ in the estimation of a single tone. In fact, on the one hand, the CLEAN and RELAX algorithms rely on the coarse estimate generated by the periodogram method for each detected tone and, eventually, exploit zero-padding to improve spectral resolution. On the other hand, the $\mathrm{Alg}-\mathrm{M}$, the Alg-YA, the Alg-S and the CFH algorithm compute the frequency residual by means of open-loop interpolation or iterative methods; the last methods refine the estimate of the frequency residual through multiple iterations. All the single tone estimators employed in these algorithms differ from the ones used in the SFEC and CSFEC, and their hybrid versions.

Use of a re-estimation procedure - In the CLEAN and RELAX algorithms and in Alg-M, once a new tone has been estimated, all the previously computed tones are re-estimated by subtracting the contribution of all the other tones; tone cancellation is accomplished in the time domain in the CLEAN and RELAX algorithms, whereas is carried out in the frequency domain in the Alg-M. The last approach is also adopted in our estimation algorithms. Finally, the CFH algorithm, the Alg-S and the Alg-YA accomplish re-estimation after computing a coarse estimate of all the parameters of the detected tones. However, the $\mathrm{CFH}$ algorithm executes this only once, whereas the Alg-S and the Alg-YA repeat it a given number of times.

Use of oversampling - The use of oversampling is proposed for the CLEAN, the RELAX, the Alg-S, and the SFEC (CSFEC) and the HSFEC (HCSFEC) algorithms only.

\section{E. Computational complexity}

The complexity of the estimation algorithms developed in Paragraphs III-A-III-C has been carefully assessed in terms of number of floating operations (flops) to be executed in the detection of $L$ targets. The general criteria adopted in estimating the overall computational cost of the SFE and that of the CSFE algorithms ${ }^{4}$ are summarised in Appendix C, where a detailed

\footnotetext{
${ }^{4}$ Note that the computational costs of the CSDE (and, similarly, of the CSDEC) are equal to that of the CSFE (of the CSFEC), respectively.
} 


\begin{tabular}{lc}
\hline Algorithm & $\mathcal{O}(\cdot)$ \\
\hline CLEAN & $\mathcal{O}\left(L^{2} N_{0} \log _{2}\left(N_{0}\right)\right)$ \\
RELAX & $\mathcal{O}\left(L^{2} N_{0} \log _{2}\left(N_{0}\right)\right)$ \\
Alg-M & $\mathcal{O}\left(N_{0} \log _{2}\left(N_{0}\right)+L^{2} K_{M}\right)$ \\
Alg-YA & $\mathcal{O}\left(L N \log _{2}(N)+L Q N\right)$ \\
CFH & $\mathcal{O}\left(L N \log _{2}(N)\right)$ \\
Alg-S & $O\left(L N \log _{2}(N)+L Q N\right)$ \\
\hline
\end{tabular}

TABLE I: Order of the computational complexity of various estimation algorithms.

analysis of the contributions due to the different tasks accomplished by each of them is also provided. Our analysis leads to the conclusion that these are approximately of order $\mathcal{O}\left(M_{\mathrm{SFE}}\right)$ and $\mathcal{O}\left(M_{\mathrm{CSFE}}\right)$, respectively, with

$$
M_{\mathrm{SFE}}=N_{0} \log _{2} N_{0}+K_{\mathrm{SFE}} N_{\mathrm{SFE}} I^{2}
$$

and

$$
M_{\mathrm{CSFE}}=N_{0} \log _{2} N_{0}+K_{\mathrm{CSFE}} N_{\mathrm{CSFE}} I^{2} ;
$$

here, $N_{\mathrm{SFE}}\left(N_{\mathrm{CSFE}}\right)$ represents the overall number iterations accomplished by the SFE (CSFE), $K_{\mathrm{SFE}}=2 / 3$ and $K_{\mathrm{CSFE}}=$ $1 / 2$. Our computer simulations have evidenced that, in the scenarios we considered, a small value of $I$ is required if the so called barycentric interpolation is employed (see ref. [70]). For this reason, the contribution of the second term appearing in the RHS of both eqs. (120)-(121) can be neglected, so that the order of the overall computational cost of both estimators is approximated well by the first term, i.e. from the term that originates from FFT processing. Moreover, based on the last results, it is not difficult to show that the computational costs of the SFEC and CSFEC are approximately of order $\mathcal{O}\left(M_{\mathrm{SFEC}}\right)$ and $\mathcal{O}\left(M_{\mathrm{CSFEC}}\right)$, respectively, with

$$
M_{\mathrm{SFEC}}=N_{0} \log _{2}\left(N_{0}\right)+K_{\mathrm{SFE}} L N_{\mathrm{SFE}} I^{2}
$$

and

$$
M_{\mathrm{CSFEC}}=N_{0} \log _{2}\left(N_{0}\right)+K_{\mathrm{CSFE}} L N_{\mathrm{CSFE}} I^{2},
$$

if no re-estimation is accomplished (see CSFEC-S3 in the description of the CSFEC algorithms) and the algorithms stop after detecting the last tone. Note that the first term appearing in the RHS of both eqs. (122)-(123) accounts for the initialization (and, in particular, for the computation of the vectors $\mathbf{X}_{0}(42)$ and $\left\{\mathbf{X}_{k}\right\}$ (65)), whereas the second term for the fact that, in the SFEC (CSFEC), the SFE (CSFE) is executed $L$ times. It is worth noting that the costs due to the evaluation of the estimated tones detected after the first one and to their frequency domain cancellation do not play an important role in this case. However, if re-estimation is accomplished, the parameter $L$ appearing in the RHS of (122)-(123) is replaced by $L^{2}$, since this task involves all the estimated tones. Despite this, the increase in the overall computational cost of the CSFEC (SFEC) with respect to the CSFE (SFE) is limited since, as evidenced by our simulation results, the use of re-estimation allows these algorithms to achieve convergence with a smaller value of the parameter $N_{\mathrm{SFE}}\left(N_{\mathrm{CSFE}}\right)$.

Finally, it is important to compare the computational cost of the SFEC and CSFEC algorithms with that of the CLEAN, RELAX, Alg-M, Alg-YA, CFH and Alg-S techniques considered in the previous paragraph. Their order of complexity is listed in Table I, from which the following considerations can be easily inferred:

1) The CLEAN and the RELAX algorithm are characterized by the same order, expressed by the complexity of a zero-padded FFT multiplied by $L^{2}$; the last factor is due to the fact that tone re-estimation is employed in both algorithms.

2) The Alg-M is characterized by the lowest computational cost; in fact, since it performs the cancellation of the detected tones directly in the frequency domain, tone estimation does not require the computation of additional FFTs. Moreover, since tone re-estimation is employed, the cost for the estimation of a single tone (i.e., the parameter $K_{M}$ ) is multiplied by $L^{2}$.

3) The order of complexity of the Alg-YA and the Alg-S, and that of the CFH algorithm depend on the fact that a FFT is computed for each tone; morover, an additional term equal to $L Q N$ is included in the order of the Alg-YA and the Alg-S, since these estimate all the tones $Q$ times.

4) The order of the cost of the SFEC (CSFEC) algorithm is similar to that of the Alg-M; however, in this case, three (four) FFTs are computed in the initialization phase and the cost for the estimation of each tone is multiplied by $N_{\mathrm{SFE}}\left(N_{\mathrm{CSFE}}\right)$ since tone refinement is performed within each recursion.

\section{NumericAl RESULtS}

In this section, we compare, in terms of accuracy and probability of failure $\left(P_{f}\right)$, our single frequency estimator (CSFE) with the A\&M [40], QSE and HAQSE algorithms [52], and our multiple tone estimators (CSFEC and HCSFEC algorithms) with the CFH algorithm [51], the Alg-YA [49], [50] and the Alg-S [69]. As far as the A\&M algorithm is concerned, two versions of it are considered; such versions are denoted $\mathrm{A} \& \mathrm{M} \# 1$ and $\mathrm{A} \& \mathrm{M} \# 2$ in the following and correspond to the Alg-1 and Alg-2, 
respectively, described in ref. [40]. Our numerical results are divided in two parts. The first part refers to the results generated on the basis of synthetically generated measurements, whereas the second one to the results generated on the basis of the measurements acquired through two commercial colocated MIMO radars. It is assumed that, in all the considered scenarios, a complex measurement sequence is observed; for this reason, the complex version of our algorithms is always taken into consideration.

\section{A. Numerical results based on synthetically generated measurements}

Four different scenarios have been considered in our computer simulations. In all of them, $N=512$ and $f_{s}=N \mathrm{~Hz}$ have been selected for the overall number of samples of the sequence $\left\{x_{c, n}\right\}$ and the sampling frequency, respectively; moreover, for any $L$, the phases of the $L$ tones have been randomly selected over the interval $[0,2 \pi]$, each independently of all the other ones. The specific features of these scenarios can be summarised as follows:

Scenario \#1 (S1) - This is characterized by $L=1$, i.e. by a single tone, having amplitude equal to one and whose normalised frequency is uniformly distributed over the interval $[8 / N, 28 / N]$.

Scenario \#2 (S2) - This is characterized by $L=2$, i.e. by a couple of tones, both having amplitude equal to one. Moreover, the normalised frequency $F_{0}$ of the first tone is uniformly distributed over the interval $[8 / N, 28 / N]$, whereas that of the second one is $F_{1}=F_{0}+1.1 / N$.

Scenario \#3 (S3) - This is characterized by $L \in\{2,3, \ldots, 10\}$, i.e. by a varying number of tones. The amplitude and the frequency of the $k$-th tone are given by

$$
a_{k} \triangleq 10^{-k \Delta_{a} / 10}
$$

and

$$
F_{k} \triangleq F_{0}+1.5 k / N
$$

respectively, with $k=0,1, \ldots, L-1$; here, $\Delta_{a}=2.5 / 3$ and $F_{0}$ is uniformly distributed over the interval $[8 / N, 28 / N]$.

Scenario \#4 (S4) - This is characterized by $L=10$ tones, whose amplitudes follow the law expressed by eq. (124). Moreover, the normalized frequency of the $k$-th tone is

$$
F_{k, m} \triangleq F_{0}+k \Delta F_{m}
$$

with $F_{0}=8.3 / N$ and $k=0,1, \ldots, 9$; here,

$$
\Delta F_{m}=(1.5+0.2 m) / N
$$

represents the normalized frequency spacing between adjacent tones and is controlled through the non negative parameter $m$ ( $m=0,1, \ldots, 5$ is assumed in the following).

In our computer simulations, the following performance indices have been assessed for each of the considered algorithms: a) the root mean square error (RMSE) for the normalized frequency estimates $\left(\mathrm{RMSE}_{f}\right)$ and for the phase estimates $\left(\mathrm{RMSE}_{p}\right)$; b) the failure probability $\left(P_{f}\right)$, i.e. the probability that the considered algorithm does not converge. It is important to point out that: 1) in our simulation, a failure event is detected when the absolute value of the normalised frequency error exceeds the threshold $\Delta \varepsilon_{F} \triangleq 1 /\left(2 N_{0}\right)$ (i.e., the size of half the frequency bin characterizing FFT processing); 2) the simulation runs in which an algorithm fails are ignored in the evaluation of its RMSEs; 3 ) each value of the three perfomance indices defined above is computed by running 20000 Monte-Carlo simulations.

The performance of five single tone estimators, namely the CSFE, A\&M\#1, A\&M\#2, QSE and HAQSE algorithms, has been assessed in S1. The following parameters have been selected for them: a) overall number of iterations $N_{\mathrm{CSFE}}=20$ and interpolation $\operatorname{order}^{5} I=7$ for the CSFE; b) overall number of iterations $N_{\mathrm{A} \& \mathrm{M}}=2$ for the two A\&M algorithms; c) $N_{\mathrm{QSE}}=3$ for the QSE algorithm; d) $N_{\mathrm{HAQSE}}=2$ for the HAQSE algorithm. In addition, the parameter $q$ of the QSE and HAQSE algorithms has been evaluated on the basis of ref. [52, eq. (39)] and the oversampling factor $M=1$ has been selected for all the considered algorithms. Our numerical results have evidenced that: 1) all these algorithms exhibit a similar dependence of $\mathrm{RMSE}_{f}$ and $\mathrm{RMSE}_{p}$ on the

$$
\mathrm{SNR} \triangleq 1 / \sigma^{2}
$$

and their accuracy approaches the Cramer-Rao lower bound (CLRB) [71] both in frequency and in phase estimation for SNR $\in[-15,20] \mathrm{dB} ; 2)$ they are characterized by a similar probability of failure; 3 ) the CSFE is characterized by a lower convergence speed than the other algorithms. As far as the last point is concerned, it is worth mentioning that: 1) the A\&M\#1, A\&M\#2, QSE and HAQSE algorithms usually require 2-4 iterations to achieve convergence, whereas the overall number of iterations required by the CSFE is 3-4 times larger; 2) the A\&M\#1, A\&M\#2, QSE and HAQSE algorithms are characterised by similar computation time, whereas the CSFE is about 10 times slower.

Some numerical results referring to $\mathbf{S 2}$ are illustrated in Figs. 3, 4 and 5. More specifically, the dependence of RMSE $f$ and $\mathrm{RMSE}_{p}$ on the average $\mathrm{SNR}^{6}$ is represented in Figs. 3 and 4, respectively, whereas the dependence of $P_{f}$ on the SNR

\footnotetext{
${ }^{5}$ In all our simulations, the barycentric interpolation described in ref. [70] has been always used.

${ }^{6}$ Since the amplitudes of both tones are equal to one, the SNR is still computed on the basis of eq. (128).
} 


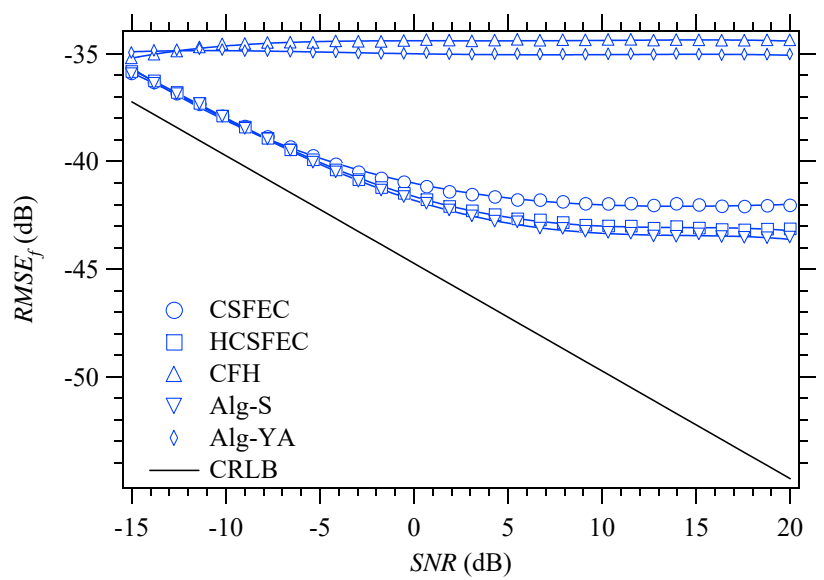

Fig. 3: RMSE performance achieved in frequency estimation versus SNR (second scenario). The Alg-S, the Alg-YA, and the CSFEC, HCSFEC and CFH algorithms are considered. The CRLB is also shown for comparison.

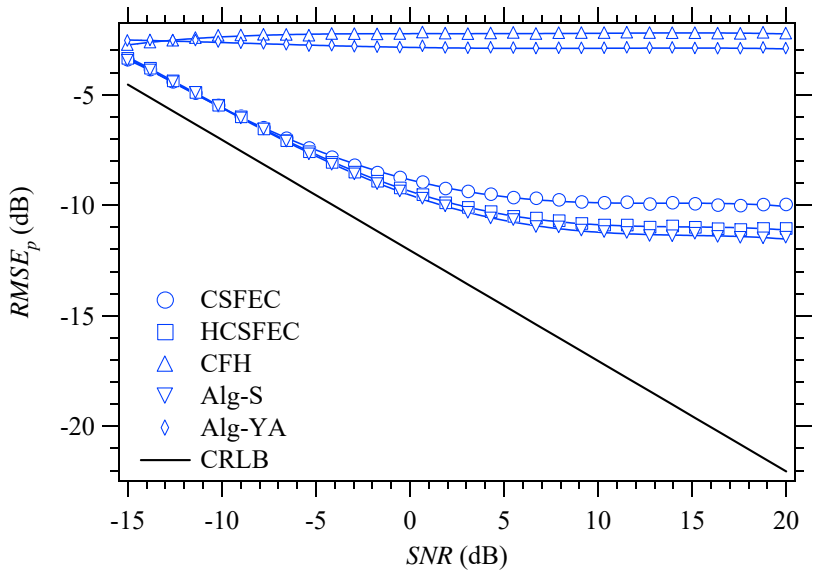

Fig. 4: RMSE performance achieved in phase estimation versus SNR (second scenario). The Alg-S, the Alg-YA, and the CSFEC, HCSFEC and CFH algorithms are considered. The CRLB is also shown for comparison.

is illustrated in Fig. 5 for all the considered algorithms; once again, the SNR range $[-15,20] \mathrm{dB}$ has been considered. The accuracy of five multiple tone estimators, namely the Alg-YA, the Alg-S, and the CSFEC, HCSFEC and CFH algorithms has been assessed in this case; moreover, the following parameters have been selected for these algorithms in S2 and S3: a) overall number of iterations $N_{\mathrm{CSFE}}=15$, number of re-estimations $N_{\mathrm{RES}}=5$ and interpolation order $I=7$ for the CSFEC; b) $N_{\mathrm{CSFE}}=5, N_{\mathrm{RES}}=1, I=7$ and number of Alg-YA iterations $N_{Y A}=5$ for the HCSFEC; c) the same parameters as the HAQSE in $\mathbf{S 1}$ for the CFH algorithm; d) overall number of iterations $Q=2$ for the Alg-YA. Moreover, the number of re-estimations carried out by the Alg-S has been evaluated on the basis of ref. [69, eq. (33)]. Our results show that: 1) the $\mathrm{CFH}$ algorithm and the Alg-YA are outperformed by the other estimation techniques for any value of SNR both in frequency and phase estimation; 2) the trend in the accuracy of the Alg-S, the CSFEC and the HCSFEC algorithms diverges from that of the CRLB for SNR $>-6 \mathrm{~dB} ; 3$ ) the CSFEC is slightly outperformed by the Alg-S and the CSFEC algorithm for SNR $>-6$ $\mathrm{dB} ; 4)$ the CSFEC and the HCSFEC techniques are more robust than all the other estimators, since their probability of failure is substantially lower for SNR $>-10$. It is also worth mentioning that the CFH algorithm and the Alg-YA require similar computation times, whereas the HCSFEC algorithm and the Alg-S (the CSFEC algorithm) are about 2 (16) times slower.

Our simulations referring to $\mathbf{S 3}$ have allowed us to assess how the performance of the considered algorithms is influenced by the overall number of tones. Some results referring to this scenario are shown in Fig. 6, which shows the dependence of $P_{f}$ on $L$; an $\mathrm{SNR}=10 \mathrm{~dB}$ for the strongest tone, and $M=1$ and 2 are assumed. From this figure it is easily inferred that: 1) the CFH algorithm, and the Alg-YA and the Alg-S have very high failure probability for $L \geq 7 ; 2$ ) the CSFEC and the HCSFEC algorithms are substantially more robust than the CFH algorithm, the Alg-YA and the Alg-S (the Alg-S), since their failure probability is lower than that of the other algorithms for $L \geq 6$ (for any $L$ ) if $M=1(M=2$ ); 3) the CFH algorithm performs slightly better than the CSFEC and the HCSFEC algorithms for $L=4$ and $M=1 ; 4$ ) the CSFEC and the HCSFEC techniques achieve a similar probability of failure. Once again, the price to be paid for a lower failure probability is represented by a larger computational effort. For instance, if $L=6$ and $M=1$, the computation time required by the CSFEC 


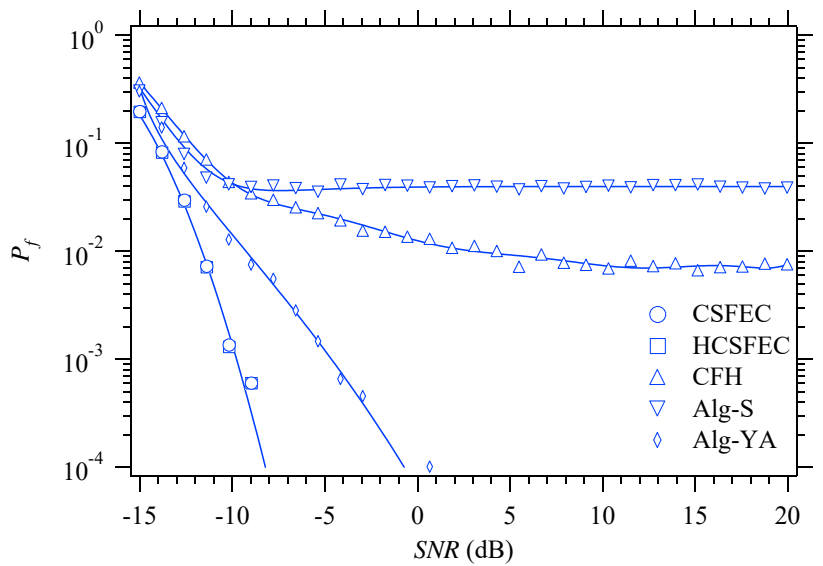

Fig. 5: Probability of failure versus SNR (second scenario). The Alg-S, the Alg-YA, and the CSFEC, HCSFEC and CFH algorithms are considered.

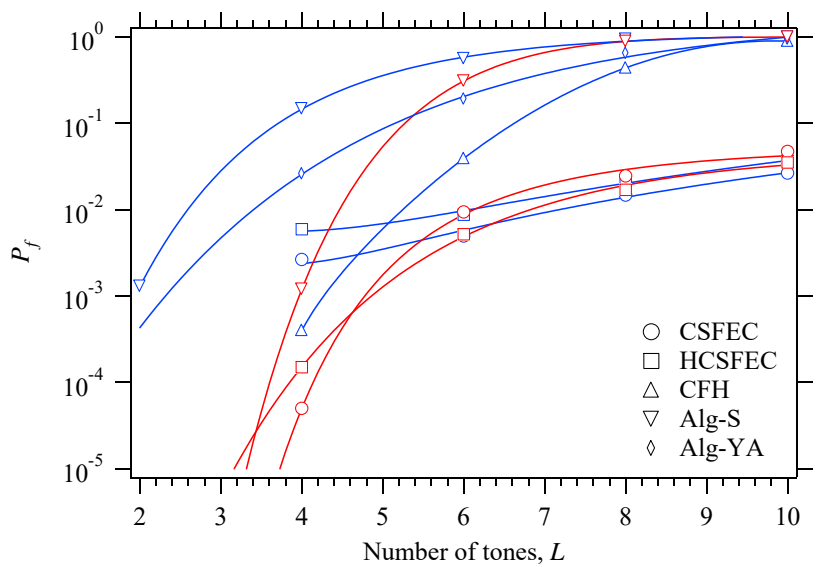

Fig. 6: Probability of failure versus number of tones (third scenario). The Alg-S, the Alg-YA, and the CSFEC, HCSFEC and CFH algorithms are considered; moreover, $M=1$ (blue curves) and 2 (red curves) are assumed.

(HCSFEC) algorithm is about 50 (4) times larger than that characterizing the CFH algorithm; if, instead, $L=4$ and $M=2$, the computation time required by the CSFEC (HCSFEC) algorithm is about 10 (1.7) times larger than that characterizing the Alg-S.

Finally, our analysis of $\mathbf{S 4}$ has allowed us to assess how different estimators perform in the presence of many closely spaced tones, having different strengths. The dependence of $\mathrm{RMSE}_{f}$, evaluated for the each of the normalized frequencies, on the tone spacing $\Delta F \cdot N$ is shown in Fig. 7, whose results have been obtained under the assumption that $\mathrm{SNR}=10 \mathrm{~dB}$ for the strongest tone (i.e., for the tone having the smallest frequency; see eq. (124)). The algorithms considered in this case are the CSFEC and the HCSFEC only (with $M=4$ ), since the $P_{f}$ of the CFH algorithm, the Alg-YA and the Alg-S is very high (see Fig. 6). Moreover, the following parameters have been selected for these algorithms a) $N_{\mathrm{CSFE}}=5, N_{\mathrm{RES}}=1$ and $I=7$ for the CSFEC and the HCSFEC; b) $N_{Y A}=3$ for the HCSFEC. The results shown in Fig. 7 lead easily to the following conclusions: 1) the $\mathrm{RMSE}_{f}$ characterizing the HCSFEC is lower than that achieved by the CSFEC for any value of tone separation and for any tone, since the CSFEC technique suffers from the error introduced by its frequency bias; 2) a larger $\mathrm{RMSE}_{f}$ is observed for weaker tones at an arbitrary $\Delta F ; 3$ ) the $\mathrm{RMSE}_{f}$ characterizing each tone reaches a floor for $\Delta F>1.9 / N$.

\section{B. Experimental Results about MIMO Radar Systems}

A measurement campaign has been accomplished in the building of our institution to acquire a data set through a FMCW MIMO radar and a SFCW MIMO radar, both operating in the E-band. The first device is the TIDEP-01012 Cascade mmWave radar; it is manufactured by Texas Instrument Inc. [72] and is classified as a long range radar (LLR). Its main parameters are: a) chirp slope $\mu=4 \cdot 10^{13} \mathrm{~Hz} / \mathrm{s}$; b) bandwidth $B=2.5 \mathrm{GHz}$; c) central frequency $f_{0}=77 \mathrm{GHz}$; d) sampling frequency $f_{s}=8 \mathrm{MHz}$; e) number of samples per chirp $N=512$. Moreover, it is endowed with a planar array made of $N_{T}=12 \mathrm{TX}$ antennas and $N_{R}=16 \mathrm{RX}$ antennas; each of its antennas consists of an array of four patch antennas. In principle, $12 \cdot 16=192$ virtual antennas are available in this case; however, only 16 horizontal-aligned and equally-spaced virtual antennas, forming 


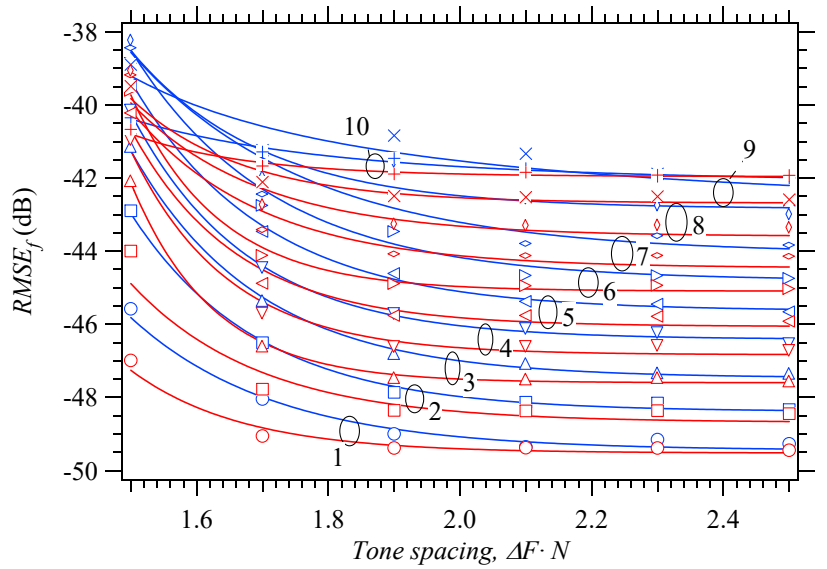

Fig. 7: RMSE performance achieved in frequency estimation versus tone separation (fourth scenario). The CSFEC (blue curves) and HCSFEC (red curves) algorithms are considered. Different tones are identified by distinct markers and numbers.

a virtual ULA with $d_{H}=\lambda / 4$, have been exploited in our work. This choice allows us to achieve the range resolution $\Delta R_{1}=c /(2 B)=5.8 \mathrm{~cm}$ and the azimuthal resolution $\Delta \theta_{1}=1.35^{\circ}$.

The second device is the Vito-In-Car radar; it is manufactured by Vayyar Imaging Ltd Company [73] and is classified as a short range radar (SRR). Its main parameters are: a) initial transmit frequency $f_{0}=78 \mathrm{GHz}$; b) frequency spacing $\Delta f=16.67$ $\mathrm{MHz}$; c) overall number of frequencies $N_{f}=121$. Therefore, the bandwidth and the central frequency of the signal it radiates are $B=2.0 \mathrm{GHz}$ and $f_{c}=79 \mathrm{GHz}$, respectively. This device is equipped with a uniform rectangular array (URA) composed by $N_{T}=16 \mathrm{TX}$ antennas and $N_{R}=21 \mathrm{RX}$ antennas, so that $16 \cdot 21=336$ virtual channels are available. However, in our work, only 16 horizontal aligned and equally spaced virtual channels, forming a virtual ULA with $d_{H}=\lambda / 4$, have been exploited. This choice allows us to achieve the range resolution $\Delta R_{2}=c /(2 B)=7.5 \mathrm{~cm}$ and the azimuthal resolution $\Delta \theta_{2}=7.6$.

All the measurements have been acquired through our radar devices in a large empty room (whose width, depth and height are $10 \mathrm{~m}, 8 \mathrm{~m}$ and $2.5 \mathrm{~m}$, respectively) under the following experimental conditions:

a) The two radar devices have been mounted on an horizontal wooden bar and have been lifted by a tripod at an height of roughly $1.60 \mathrm{~m}$ from ground (see Fig. 8); moreover, they have been activated alternately to avoid mutual interference.

b) The measurements acquired through the SFCW MIMO radar have been always pre-processed by the cancellation algorithm already available on this device; this algorithm exploits the measurements acquired from the first transmitted frame to cancel out unwanted received echoes.

c) As already mentioned above, a small virtual ULA, consisting of $N_{V}=16$ virtual antennas, has been exploited in both devices. This has allowed us to reduce the far-field distance, which is proportional to the square of the overall size of the employed ULA.

In our experimental set-up, a pico-flexx camera manufactured by PMD Technologies Inc. [74] has been employed as a reference sensor; this device is based on a near-infrared vertical cavity surface emitting laser (VCSEL), and is able to provide a depth map or, equivalently, a three-dimensional point-cloud of a small region of the observed environment (its maximum depth is equal to $4 \mathrm{~m}$, whereas its field of view is $62^{\circ} \times 45^{\circ}$ ).

As far as the acquired measurements are concerned, it is important to point out that:

a) In both the FMCW and SFCW radar systems, all the target ranges have been estimated with respect to the central virtual channel of their ULA.

b) The exact target positions have been acquired with respect to the centre of the pico-flexx camera. Therefore, in comparing these positions with their estimates computed on the basis of the radar measurements, the distance $\Delta_{F P}=50 \mathrm{~cm}\left(\Delta_{S P}=20\right.$ $\mathrm{cm}$ ) between the FMCW (SFCW) radar and the camera was always kept into account.

c) All our measurements have been processed in the MATLAB environment. A desktop computer equipped with a single i7 processor inside has been always used.

The numerical results illustrated in this paragraph refer to two specific static scenarios. The first scenario is characterized by a single detectable target, represented by a small metal disk ${ }^{7}$ of size $5.5 \mathrm{~cm}$. The target range $R$ has been varied in the interval $(1.0 \mathrm{~m}, 3.0 \mathrm{~m})$, with a step of $0.5 \mathrm{~m}$; in all cases, its azimuth $\theta$ has remained within the interval $\left(-40^{\circ}, 40^{\circ}\right)$. The exact positions considered for our target are listed in Table II for both the FMCW and the SFCW MIMO radar systems (the data referring to the $i$-th position are collected in the column identified by $T_{i}$, with $\left.i=1,2, \ldots, 10\right)$. The second scenario, instead, is characterized by the presence of an overall number of targets ranging from 1 to 5 (so that $1 \leq L \leq 5$ ). As shown in Fig. 8, the targets are represented by small coins with a diameter of $2 \mathrm{~cm}$, whose exact positions are listed in Table III

\footnotetext{
${ }^{7}$ Each target is hung from the ceiling: a nylon thread has been used for suspending it.
} 


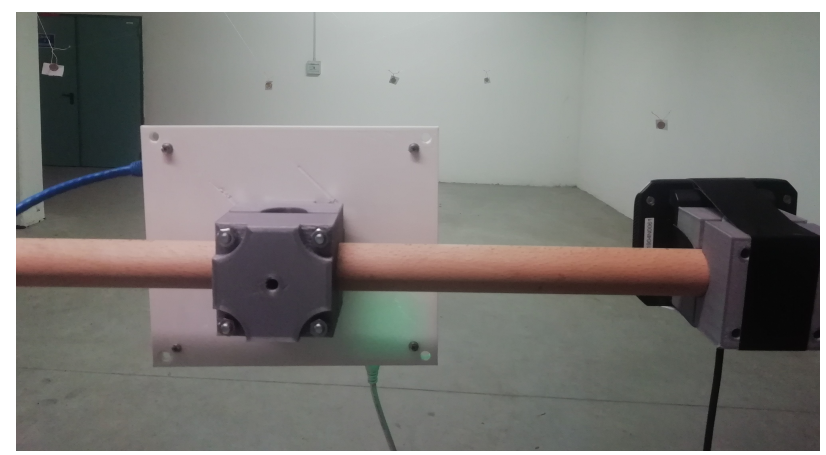

Fig. 8: Experimental set-up developed for our acquisitions. The radars are mounted on a wooden bar, whereas the targets are small coins in this case (second scenario).

(the data referring to the $i$-th target are collected in the column identified by $T_{i}$, with $i=1,2, \ldots, 5$ ); they been sequentially added in our scenario, starting from a single target and then increasing the number of targets up to 5 . This has allowed us to assess how the performance of our estimation algorithms is influenced by the value of the parameter $L$. In processing all the acquired measurements, prior knowledge of $L$ has been always assumed and the following values have been selected for the parameters of the CSFE (CSDE) and CSFEC (CSDEC) algorithms: $N_{0}=2048\left(N_{0}=1936\right), M=4(M=16)$ and $N_{\mathrm{CSFE}}=20\left(N_{\mathrm{CSDE}}=20\right)$ and $I=7$.

Moreover, in analysing the data acquired in both scenarios, the following choices have been made:

1) The accuracy of range estimates has been assessed by evaluating the RMSE

$$
\bar{\varepsilon}_{R} \triangleq \sqrt{\sum_{l=0}^{N_{m}-1}\left[R_{l}-\hat{R}_{l}\right]^{2}}
$$

and the peak error

$$
\hat{\varepsilon}_{R} \triangleq \max _{l}\left|R_{l}-\hat{R}_{l}\right|,
$$

where $N_{m}$ represents the overall number of available measurements.

2) Since the RCS of the considered targets was unknown, our analysis of the complex gains available over the 16 channels of the considered virtual ULA and associated with the same target has concerned only their (unwrapped) phase. The phases estimated by the CSFE (CSDE) over the ULA and associated with a target placed at approximately ${ }^{8} \theta=-14^{\circ}$ with respect to the center of the two radars is shown in Fig. 9. Since the distance $d_{H}$ between adjacent virtual channels is constant, the estimated phases exhibit a linear dependence on the index of the virtual channel (see eqs. (13) and (14)). Moreover, if a linear fitting is drawn for these data, it should be expected that the slope of the resulting straight line is proportional to $\sin (\theta)$ (see eq. (25)); this is confirmed by the results shown in Fig. 9 for both the FMCW and the SFCW radar systems. To assess the quality of the estimated phases, their $\operatorname{RMSE}^{9} \bar{\varepsilon}_{\psi}$ has been evaluated in both scenarios; in doing so, the linear fitting of the phases estimated over the whole ULA has been taken as a reference with respect to which each phase error has been computed.

The estimate of the target range generated by the CSFE and the CSDE in each of the $N_{m}=10$ distinct positions considered in the first scenario are listed in Table II; in the same table, the value of $\bar{\varepsilon}_{\psi}$ computed for each position is also provided . These results have allowed us to compute the errors $\bar{\varepsilon}_{R}$ (129) and $\hat{\varepsilon}_{R}$ (130), the mean of $\bar{\varepsilon}_{\psi}$ (denoted $\bar{\varepsilon}_{m, \psi}$ and generated by taking the average of the $N_{m}$ values available for $\bar{\varepsilon}_{\psi}$ ) and the average computation time (CT); their values are summarized in Table IV for the same scenario.

These results and those listed in Table II have led us to the following conclusions:

1) Both the CSFE and CSDE are able to accurately estimate the range and the amplitudes of a single target.

2) The value of $\bar{\varepsilon}_{\psi}$ characterizing the CSDE algorithm is lower when the target is closer to the radar, whereas the opposite occurs in the case of the CSFE. This result is mainly due to the fact: a) since the antenna array of the SFCW radar is more compact than that of the FMCW radar, the far-field condition of the former device is satisfied at shorter ranges than the second one: b) the SFCW (FMCW) radar is a SRR (LRR). In fact, for these reasons, the measurements provided by the SFCW radar allow to obtain more accurate results at a shorter distance. On the contrary, the higher transmit power radiated by the FMCW allows to achieve a smaller $\bar{\varepsilon}_{\psi}$ at larger ranges.

3) All the values of $\bar{\varepsilon}_{R}$ and $\hat{\varepsilon}_{R}$ are comparable, reasonably low and in the order of the resolution of our devices.

4) The value of $\bar{\varepsilon}_{m, \psi}$ achieved with the SFCW radar is slightly lower that obtained with the FMCW radar.

\footnotetext{
${ }^{8}$ The exact coordinates can be found in the column labelled by $T_{7}$ in Table II.

${ }^{9}$ The evaluation of this RMSE is based on a formula similar to eq. (129).
} 


\begin{tabular}{|c|c|c|c|c|c|c|c|c|c|c|c|}
\hline Metho & & $T_{1}$ & $T_{2}$ & $T_{3}$ & $T_{4}$ & $T_{5}$ & $T_{6}$ & $T_{7}$ & $T_{8}$ & $T_{9}$ & $T_{10}$ \\
\hline \multirow{2}{*}{ Exact (FMCW) } & $R(\mathrm{~m})$ & 1.2 & 1.25 & 1.5 & 1.6 & 2.16 & 2.23 & 2.85 & 2.55 & 2.96 & 3.25 \\
\hline & $\theta\left({ }^{\circ}\right)$ & -25 & 40 & -10 & 30 & -18 & 24 & -14 & 21 & -18 & 25 \\
\hline \multirow{2}{*}{ CSFE } & $\hat{R}(\mathrm{~m})$ & 1.166 & 1.219 & 1.523 & 1.643 & 2.184 & 2.278 & 2.885 & 2.582 & 2.932 & 3.267 \\
\hline & $\bar{\varepsilon}_{\psi}(\mathrm{rad})$ & 0.8 & 0.597 & 0.592 & 0.5 & 0.521 & 0.524 & 0.576 & 0.574 & 0.513 & 0.506 \\
\hline \multirow{2}{*}{ Exact (SFCW) } & $R(\mathrm{~m})$ & 1.2 & 1.08 & 1.5 & 1.42 & 2.05 & 2.1 & 2.75 & 2.45 & 2.88 & 3.09 \\
\hline & $\theta\left(^{\circ}\right)$ & -35 & 30 & -20 & 20 & -12 & 15 & -14 & 15 & -18 & 25 \\
\hline \multirow{2}{*}{ CSDE } & $\hat{R}(\mathrm{~m})$ & 1.242 & 1.06 & 1.517 & 1.413 & 2.025 & 2.06 & 2.78 & 2.409 & 2.905 & 3.041 \\
\hline & $\bar{\varepsilon}_{\psi}(\mathrm{rad})$ & 0.25 & 0.35 & 0.67 & 0.345 & 0.422 & 0.45 & 0.686 & 0.919 & 0.379 & 0.54 \\
\hline
\end{tabular}

TABLE II: Exact positions (range and azimuth) of the considered target (first scenario), estimated ranges and RMSE evaluated for the phase fitting over the considered sixteen virtual channels. Both CSFE and CSDE are considered.

\begin{tabular}{cccccc}
\hline Method & $\begin{array}{c}T_{1} \\
(\mathrm{~m})\end{array}$ & $\begin{array}{c}T_{2} \\
(\mathrm{~m})\end{array}$ & $\begin{array}{c}T_{3} \\
(\mathrm{~m})\end{array}$ & $\begin{array}{c}T_{4} \\
(\mathrm{~m})\end{array}$ & $\begin{array}{c}T_{5} \\
(\mathrm{~m})\end{array}$ \\
\hline Exact $(\mathrm{FMCW})$ & 2.8 & 1.95 & 2.3 & 3.87 & 2.1 \\
\hline Exact $(\mathrm{SFCW})$ & 3.2 & 2.15 & 1.85 & 3.75 & 2.45 \\
\hline CSFEC & 2.768 & 1.999 & 2.317 & 3.904 & 2.138 \\
\hline HCSFEC & 2.768 & 1.999 & 2.317 & 3.903 & 2.138 \\
\hline CSDEC & 3.08 & 2.159 & 1.75 & 3.72 & 2.456 \\
\hline HCSDEC & 3.09 & 2.163 & 1.75 & 3.73 & 2.459 \\
\hline CFH & 2.768 & 2.001 & 2.314 & 3.868 & - \\
\hline HAQSE & 2.768 & 2.003 & 2.317 & 3.868 & - \\
\hline Alg-YA & 2.768 & 1.999 & 2.317 & 3.903 & - \\
\hline
\end{tabular}

TABLE III: Exact positions of the five targets characterizing the second scenario. The range estimates computed by the CFSEC, CSDEC, HCSFEC, HCSDEC, CFH and HAQSE algorithms, and Alg-YA are also provided.

5) The CTs are in the order of few milliseconds.

Let us focus on the second scenario now. The range estimates generated by the CSFEC, HCSFEC, CSDEC, HCSDEC, CFH and HASQE algorithms, and the Alg-YA in this scenario are listed in Table III. Note that the estimates provided for the CSFEC, HCSFEC, CSDEC and HCSDEC refer to 5 targets, whereas those provided for the CFH and HASQE algorithms, and for the Alg-YA to 4 targets only. This is due to the fact that the last three algorithms, unlike the first four, do not converge in the case of 5 targets. The errors $\bar{\varepsilon}_{R}$ and $\hat{\varepsilon}_{R}$, and the CT assessed in this case are listed in Table V. From these results it is easily inferred that:

1) The CSFEC (CSDEC) algorithm and the Alg-YA achieve better accuracy than the other algorithms. However, unlike the Alg-YA, the CSFEC (CSDEC) works properly in the presence of five targets.

2) In the considered scenario, the hybrid version of the CSFEC (CSDEC) algorithm does not provide a better accuracy than CSFEC (CSDEC); this means that estimation bias of the last algorithms does not play an important role in this case.

3) The estimated RMSEs and peak errors are in the order of the resolution of our devices, but are a little bit higher in the SFCW radar system. We believe that this is mainly due to the poorer estimation of the first target, since, in our specific experiment, the energy received from this target has been found to be lower than that coming from the other four targets. This problem is not found in the FMCW radar system, since this device, being a LRR, is able to achieve an higher SNR at its RX side for all the targets.

Finally, we would like to stress that the robustness of CSFEC and CSDEC algorithms is related to the accuracy of the estimation and cancellation procedure they accomplish. This is exemplified by Fig. 10, where the initial amplitude spectrum of the signal received on the central virtual channel of the FMCW radar system in the second scenario and its residual, resulting from the cancellation of the spectral contributions due to the five detected targets, are shown. 


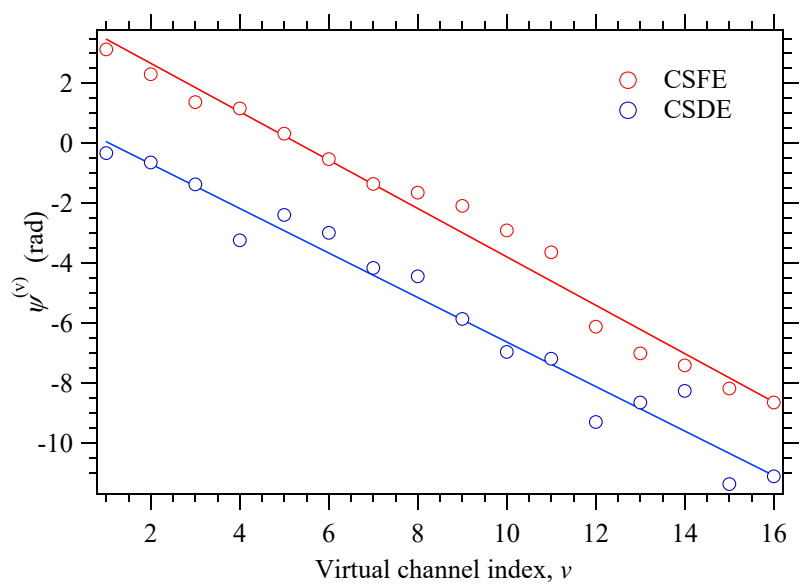

Fig. 9: Unwrapped phase of the complex gain versus index of the virtual channel of the employed ULA. A single target is assumed. The phase estimates generated by the CSFE (CSDE) are identified by red circles (red crosses), whereas the straight lines represent their linear fitting.

\begin{tabular}{lcccc}
\hline Methods & $\begin{array}{c}\bar{\varepsilon}_{R} \\
(\mathrm{~m})\end{array}$ & $\begin{array}{c}\hat{\varepsilon}_{R} \\
(\mathrm{~m})\end{array}$ & $\begin{array}{c}\bar{\varepsilon}_{m, \psi} \\
(\mathrm{rad})\end{array}$ & $\begin{array}{c}\mathrm{CT} \\
(\mathrm{msec})\end{array}$ \\
\hline CSFE & 0.033 & 0.048 & 0.570 & 1.05 \\
\hline CSDE & 0.032 & 0.049 & 0.501 & 1.01 \\
\hline
\end{tabular}

TABLE IV: Root mean square error $\bar{\varepsilon}_{R}$, peak error $\hat{\varepsilon}_{R}$, mean error $\bar{\varepsilon}_{m, \psi}$ and computation time (CT) evaluated in the first scenario.

\section{CONCLUSIONS}

In this manuscript, a novel algorithm for detecting and estimating a single tone has been derived; moreover, its has been shown how it can be exploited to estimate multiple tones through a serial cancellation procedure. The accuracy and robustness of the devised estimators has been assessed by means of extensive computer simulations and by analysing their application to range detection and phase estimation in commercial MIMO radars. Moreover, our results have evidenced that these estimators outperform all the other related estimators available in the technical literature in terms of probability of convergence and accuracy, in the presence of both a single tone and multiple tones. Future work concerns the application of the developed algorithms to other fields.

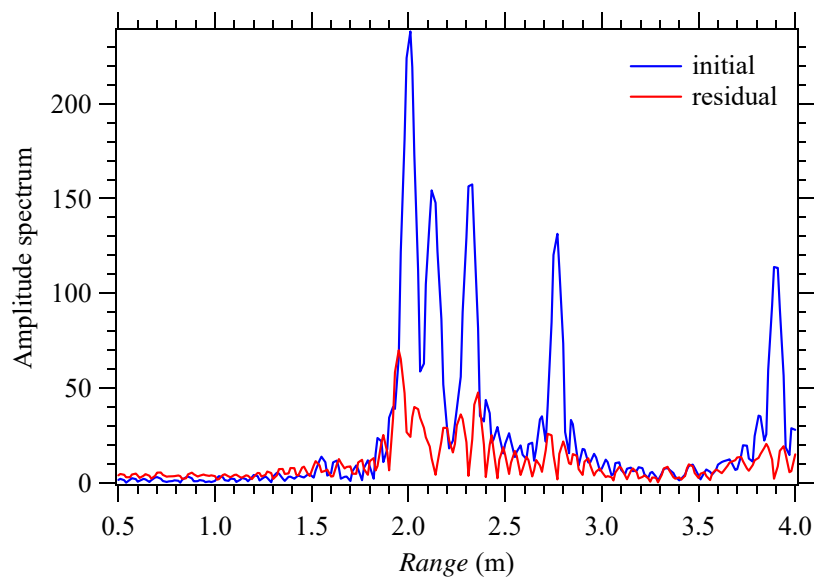

Fig. 10: Representation of the initial amplitude spectrum of the signal observed on the central virtual channel (blue line) and of the final residual amplitude spectrum generated by the CFSEC algorithm (red line). The second scenario is considered. 


\begin{tabular}{cccc}
\hline Methods & $\begin{array}{c}\bar{\varepsilon}_{R} \\
(\mathrm{~m})\end{array}$ & $\begin{array}{c}\hat{\varepsilon}_{R} \\
(\mathrm{~m})\end{array}$ & $\begin{array}{c}\mathrm{CT} \\
(\mathrm{msec})\end{array}$ \\
\hline CSFEC & 0.031 & 0.049 & 5 \\
\hline HCSFEC & 0.031 & 0.049 & 6 \\
\hline CSDEC & 0.075 & 0.110 & 5 \\
\hline HCSDEC & 0.075 & 0.110 & 6 \\
\hline CFH & 0.033 & 0.051 & 2 \\
\hline HAQSE & 0.034 & 0.053 & 1 \\
\hline Alg-YA & 0.031 & 0.049 & 10 \\
\hline
\end{tabular}

TABLE V: Root mean square error $\bar{\varepsilon}_{R}$, peak error $\hat{\varepsilon}_{R}$, and computation time (CT) evaluated in the second scenario.

\section{ACKNOWLEDGMENTS}

The authors would like to thank CNH Industrial Italia S.p.A. and CNH Industrial Belgium NV for funding this research work.

\section{APPENDIX A}

In this appendix, the derivation of the approximate $\operatorname{MSEs} \varepsilon_{\mathrm{SFE}}(\tilde{\Delta}, \hat{C})(60)$ and $\varepsilon_{\mathrm{CSFE}}(\tilde{\Delta}, \hat{A})(96)$ is sketched. To begin, we rewrite eq. (59) as

$$
\begin{aligned}
& \varepsilon_{n}(\tilde{F}, \hat{C})=x_{r, n}^{2}+2\left[\hat{C}_{R}^{2}+\hat{C}_{I}^{2}\right] \\
& -4 \hat{C}_{R}\left[x_{r, n} \cos \left(\hat{\theta}_{n}\right) \cos (n \tilde{\Delta})-x_{r, n} \sin \left(\hat{\theta}_{n}\right) \sin (n \tilde{\Delta})\right] \\
& +4 \hat{C}_{I}\left[x_{r, n} \sin \left(\hat{\theta}_{n}\right) \cos (n \tilde{\Delta})+x_{r, n} \cos \left(\hat{\theta}_{n}\right) \sin (n \tilde{\Delta})\right] \\
& +2\left(\hat{C}_{R}^{2}-\hat{C}_{I}^{2}\right)\left[\cos \left(2 \hat{\theta}_{n}\right) \cos (2 n \tilde{\Delta})-\sin \left(2 \hat{\theta}_{n}\right) \sin (2 n \tilde{\Delta})\right] \\
& -4 \hat{C}_{R} \hat{C}_{I}\left[\sin \left(2 \hat{\theta}_{n}\right) \cos (2 n \tilde{\Delta})+\cos \left(2 \hat{\theta}_{n}\right) \sin (2 n \tilde{\Delta})\right]
\end{aligned}
$$

using standard trigonometric formulas. If the normalized frequency $F_{\mathrm{DFT}}$ (41) is small enough (i.e., if the FFT order $N_{0}$ is large enough), the trigonometric functions $\cos (k n \tilde{\Delta})$ and $\sin (k n \tilde{\Delta})$ appearing in the RHS of eq. (131) (with $k=1$ and 2) can be approximated as ${ }^{10}$

$$
\begin{gathered}
\sin (n \tilde{\Delta}) \simeq n \tilde{\Delta}, \\
\cos (n \tilde{\Delta}) \simeq 1-\frac{1}{2} n^{2} \tilde{\Delta}^{2}, \\
\sin (2 n \tilde{\Delta}) \simeq 2 n \tilde{\Delta}-\frac{4}{3} n^{3} \tilde{\Delta}^{3}
\end{gathered}
$$

and

$$
\cos (2 n \tilde{\Delta}) \simeq 1-2 n^{2} \tilde{\Delta}^{2}
$$

Substituting the RHSs of eqs. (132)-(135) in that of eq. (131) and, then, substituting the resulting approximate expression in the RHS of eq. (31) yields eq. (60).

A similar procedure is followed in the derivation of eq. (96). First of all, eq. (90) is rewritten as

$$
\begin{aligned}
\varepsilon_{n}(\tilde{F}, \tilde{A}) & =\tilde{A}_{R}^{2}+\tilde{A}_{I}^{2}-2\left(x_{c, n}^{(R)} \tilde{A}_{R}+x_{c, n}^{(I)} \tilde{A}_{I}\right) \cos \left(\tilde{\phi}_{n}\right) \\
& -2\left(x_{c, n}^{(I)} \tilde{A}_{R}-x_{c, n}^{(R)} \tilde{A}_{I}\right) \sin \left(\tilde{\phi}_{n}\right)+\left|x_{c, n}\right|^{2},
\end{aligned}
$$

${ }^{10}$ Note that the approximation adopted for $\sin (2 n \tilde{\Delta})(\cos (2 n \tilde{\Delta}))$ is more accurate than the one used for $\sin (n \tilde{\Delta})(\cos (n \tilde{\Delta}))$, since the width of the interval of its argument is twice that of the last function. 
where $\tilde{A}_{R} \triangleq \Re\{\tilde{A}\}, \tilde{A}_{I} \triangleq \Im\{\tilde{A}\}, x_{c, n}^{(R)} \triangleq \Re\left\{x_{c, n}\right\}, x_{c, n}^{(I)} \triangleq \Im\left\{x_{c, n}\right\}$ and $\tilde{\phi}_{n}$ is defined by eq. (56) (see also eqs. (57)-(58)). Substituting the RHS of eq. (56) in that of eq. (136) produces, after some manipulation,

$$
\begin{aligned}
\varepsilon_{n}(\tilde{F}, \tilde{A}) & =\left|x_{c, n}\right|^{2}+\tilde{A}_{R}^{2}+\tilde{A}_{I}^{2} \\
& -2\left(x_{c, n}^{(R)} \tilde{A}_{R}+x_{c, n}^{(I)} \tilde{A}_{I}\right) \\
& \cdot\left[\cos \left(\hat{\theta}_{n}\right) \cos (n \tilde{\Delta})-\sin \left(\hat{\theta}_{n}\right) \sin (n \tilde{\Delta})\right] \\
& -2\left(x_{c, n}^{(I)} \tilde{A}_{R}-x_{c, n}^{(R)} \tilde{A}_{I}\right) \\
\cdot & {\left[\sin \left(\hat{\theta}_{n}\right) \cos (n \tilde{\Delta})+\cos \left(\hat{\theta}_{n}\right) \sin (n \tilde{\Delta})\right] . }
\end{aligned}
$$

Finally, substituting the approximations (133) and

$$
\sin (n \tilde{\Delta}) \simeq n \tilde{\Delta}-n^{3} \frac{\tilde{\Delta}^{3}}{6}
$$

in the RHS of eq. (137) and, then, the resulting expression in the RHS of eq. (92) produces eq. (96).

\section{APPENDIX B}

\section{A. Spectrum cancellation}

In this paragraph, the expression of the vector $\overline{\mathbf{C}}_{0}\left(\hat{A}_{l}[r-1], \hat{F}_{l}[r-1]\right)$ appearing in the RHS of eq. (106) is derived. First of all, let us take into consideration the CSFE; in this case, $\overline{\mathbf{C}}_{0}(\cdot, \cdot)$ is computed to cancel the contribution of the sequence (see eq. (91))

$$
s_{n}\left(\bar{F}_{l}, \bar{A}_{l}\right) \triangleq \bar{A}_{l} \exp \left(j 2 \pi n \bar{F}_{l}\right)=\bar{A}_{l} \bar{w}_{l}^{n}
$$

to the vector $\mathbf{X}_{0}$ (42); here,

$$
\bar{w}_{l} \triangleq \exp \left(j 2 \pi \bar{F}_{l}\right)
$$

Since $\mathbf{X}_{0}$ is the $N_{0}$-th order DFT of the zero-padded vector $\mathbf{x}_{0, Z P}(43)$ (where the vector $\mathbf{x}_{0}$ collects the elements of the complex sequence $\left.\left\{x_{c, n} ; n=0,1, \ldots, N-1\right\}\right)$, it is easy to prove that

$$
\overline{\mathbf{C}}_{0}\left(\hat{A}_{l}[r-1], \hat{F}_{l}[r-1]\right)=\bar{A}_{l} \quad \overline{\mathbf{W}}_{0}^{(l)},
$$

where $\overline{\mathbf{W}}_{0}^{(l)}$ denote the $N_{0}$-th order DFT of the vector

$$
\overline{\mathbf{w}}_{0}^{(l)} \triangleq\left[1, \bar{w}_{l}, \bar{w}_{l}^{2}, \ldots, \bar{w}_{l}^{N-1}, 0, \ldots, 0\right]^{T} .
$$

Then, the $m$-th element of the vector $\overline{\mathbf{W}}_{0}^{(l)}$ is given by

$$
\begin{aligned}
\bar{W}_{0}^{(l)}[m] & =\frac{1}{N} \sum_{n=0}^{N-1} \bar{w}_{l}^{n} \exp \left(-j \frac{2 \pi m}{N_{0}} n\right) \\
& =\frac{1}{N} \sum_{n=0}^{N-1}(q[m])^{n},
\end{aligned}
$$

where

$$
q[m] \triangleq \exp \left(j 2 \pi\left(\hat{F}-\frac{m}{N_{0}}\right)\right) .
$$

Therefore, the identity

$$
\sum_{n=0}^{N-1} q^{n}=\frac{q^{N}-1}{q-1}
$$

holding for any $q \in \mathbb{C}$, can be exploited for an efficient computation of all the elements of the vector $\overline{\mathbf{W}}_{0}^{(l)}$.

Similar considerations can be formulated for the SFE. However, in this case, eq. (139) is replaced by (see eq. (33))

$$
\begin{aligned}
s_{n}\left(\bar{F}_{l}, \bar{C}_{l}\right) & \triangleq \bar{C}_{l} \exp \left(j 2 \pi n \bar{F}_{l}\right)+\bar{C}_{l}^{*} \exp \left(-j 2 \pi n \bar{F}_{l}\right) \\
& =\bar{C}_{l}\left(\bar{w}_{l}\right)^{n}+\bar{C}_{l}^{*}\left(\bar{w}_{l}^{*}\right)^{n},
\end{aligned}
$$

where $\bar{w}_{l}$ is still defined by eq. (140). Consequently, eq. (141) is replaced by

$$
\overline{\mathbf{C}}_{0}\left(\hat{A}_{l}[r-1], \hat{F}_{l}[r-1]\right)=\bar{C}_{l} \overline{\mathbf{W}}_{0}^{(l)}+\bar{C}_{l}^{*} \overline{\mathbf{W}}_{0, c}^{(l)},
$$


where $\overline{\mathbf{W}}_{0}^{(l)}$ is the $N_{0}$-dimensional vector defined above and $\overline{\mathbf{W}}_{0, c}^{(l)}$ denote the $N_{0}$-th order DFT of the vector $\left(\overline{\mathbf{w}}_{0}^{(l)}\right)^{*}$. Therefore, the $m$-th element of the vector $\overline{\mathbf{W}}_{0, c}^{(l)}$ is given by

$$
\begin{aligned}
\bar{W}_{0, c}^{(l)}[m] & =\frac{1}{N} \sum_{n=0}^{N-1}\left(\bar{w}_{l}^{*}\right)^{n} \exp \left(-j \frac{2 \pi m}{N_{0}} n\right) \\
& =\frac{1}{N} \sum_{n=0}^{N-1}\left(q_{c}[m]\right)^{n},
\end{aligned}
$$

where

$$
q_{c}[m] \triangleq \exp \left(j 2 \pi\left(-\hat{F}-\frac{m}{N_{0}}\right)\right)
$$

Again, the identity (145) can be exploited for an efficient computation of all the elements of both the vectors $\overline{\mathbf{W}}_{0}^{(l)}$ and $\overline{\mathbf{W}}_{0, c}^{(l)}$.

Similar considerations can be formulated for the CSDE. However, in this case, eq. (140) is replaced by

$$
\bar{w}_{l} \triangleq \exp \left(-j 2 \pi \bar{F}_{l}\right) \text {. }
$$

Consequently, eq. (141) still holds, but has a different meaning, since $\overline{\mathbf{W}}_{0}^{(l)}$ denote the $N_{0}$-th order IDFT of the vector

$$
\overline{\mathbf{w}}_{0}^{(l)} \triangleq\left[1, \bar{w}_{l}, \bar{w}_{l}^{2}, \ldots, \bar{w}_{l}^{N-1}, 0, \ldots, 0\right]^{T} .
$$

Then, the $m$-th element of the vector $\overline{\mathbf{W}}_{0}^{(l)}$ is still expressed by the RHS of eq. (143), but

$$
q[m] \triangleq \exp \left(-j 2 \pi\left(\hat{F}-\frac{m}{N_{0}}\right)\right) .
$$

\section{B. Leakage cancellation}

In this paragraph, the expression of the quantity $\bar{X}_{\mathrm{lk}, k}\left(\hat{A}_{l}[r-1], \hat{F}_{l}[r-1], \hat{F}_{c, r}[r]\right)$ appearing in the RHS of eqs. (112) and (115) is derived for the CSFEC and the SFEC algorithms. In the first case, this quantity is computed to cancel the contribution of the sequence (139) to $\bar{X}_{k, \rho[r]}$ for any $k$. Since $\bar{X}_{k, \rho[r]}$ is defined by eq. (63), it is not difficult to show that

$$
\bar{X}_{\mathrm{lk}, k}\left(\hat{A}_{l}[r-1], \hat{F}_{l}[r-1], \hat{F}_{c, r}[r]\right)=\bar{C}_{l} \bar{W}_{k}^{(l)}\left(\hat{F}_{c, r}[r]\right),
$$

where

$$
\begin{aligned}
\bar{W}_{k}^{(l)}\left(\hat{F}_{c, r}[r]\right) & =\frac{1}{N} \sum_{n=0}^{N-1} n^{k} \bar{w}_{l}^{n} \exp \left(-j 2 \pi n \hat{F}_{c, r}[r]\right) \\
& =\frac{1}{N} \sum_{n=0}^{N-1} n^{k}\left(p^{(m)}\right)^{n}
\end{aligned}
$$

and

$$
p^{(m)} \triangleq \exp \left(j 2 \pi\left(\bar{F}_{l}-\hat{F}_{c, r}[r]\right)\right) .
$$

Therefore, the identities (145) and (84)-(85) can be exploited for an efficient computation of all the terms appearing in the RHS of eqs. (112) and (115) for $k=0,1,2$ and 3.

In the second case, the only modification with respect to the first one consists in adding the term $\bar{C}_{l} \bar{W}_{k, c}^{(l)}\left(\hat{F}_{c, r}[r]\right)$ to the RHS of eq. eq. (153). Note that

$$
\begin{aligned}
\bar{W}_{k, c}^{(l)}\left(\hat{F}_{c, r}[r]\right) & =\frac{1}{N} \sum_{n=0}^{N-1} n^{k}\left(\bar{w}_{l}^{*}\right)^{n} \exp \left(-j 2 \pi n \hat{F}_{c, r}[r]\right) \\
& =\frac{1}{N} \sum_{n=0}^{N-1} n^{k}\left(p_{c}^{(m)}\right)^{n}
\end{aligned}
$$

where

$$
p_{c}^{(m)} \triangleq \exp \left(-j 2 \pi\left(\bar{F}_{l}+\hat{F}_{c, r}[r]\right) \cdot\right)
$$

Therefore, the identities (145) and (84)-(85) can be exploited for an efficient computation of all the terms appearing in the RHS of eqs. (112) and (115) even if the SFEC algorithm is used. 
A similar procedure for leakage cancellation can be used also for the CSDEC algorithm. In this case, similarly to the CSFEC, the expression of $\bar{X}_{\mathrm{lk}, k}$ is still defined by eq. (153); however, the quantity $\bar{W}_{k}^{(l)}\left(\hat{F}_{c, r}[r]\right)$ is computed as

$$
\begin{aligned}
\bar{W}_{k}^{(l)}\left(\hat{F}_{c, r}[r]\right) & =\frac{1}{N} \sum_{n=0}^{N-1} n^{k} \bar{w}_{l}^{n} \exp \left(j 2 \pi n \hat{F}_{c, r}[r]\right) \\
& =\frac{1}{N} \sum_{n=0}^{N-1} n^{k}\left(p^{(m)}\right)^{n}
\end{aligned}
$$

with

$$
p^{(m)} \triangleq \exp \left(-j 2 \pi\left(\bar{F}_{l}-\hat{F}_{c, r}[r]\right)\right) .
$$

Therefore, the identities (145) and (84)-(85) can be exploited for an efficient computation of all the terms appearing in the RHS of eqs. (112) and (115) for $k=0,1,2$ and 3.

\section{APPENDIX C}

In this Appendix, the computational complexity, in terms of flops, is assessed for the SFE and the CSFE developed in Paragraph III-A. The overall computational cost of each these algorithms can be expressed as

$$
\mathcal{C}_{\text {alg }}=\mathcal{C}_{i, \text { alg }}+N_{\text {alg }} \mathcal{C}_{r, \text { alg }}
$$

where $\mathcal{C}_{i, \text { alg }}, \mathcal{C}_{r, \text { alg }}$ and $N_{\text {alg }}$ represent the cost of its initialization, that of each of its iterations and the overall number of iterations, respectively, and 'alg' denotes the algorithm these parameters refer to (SFE or CSFE). The general criteria adopted in estimating the computational cost of both $\mathcal{C}_{i, \text { alg }}$ and $\mathcal{C}_{r, \text { alg }}$ are the same as those illustrated in [75] and can be summarised as follows:

- $4 d-2$ flops are required to compute the inner product $\mathbf{u}_{c}^{T} \mathbf{v}$ of a $d \times 1$ complex column vector and a $d \times 1$ real column vector;

- $6 d+2(d-1)$ flops are required to compute the inner product $\mathbf{u}_{c}^{T} \mathbf{v}_{c}$ of two $d \times 1$ complex vectors;

- $d$ flops are required to find the maximum element of a vector $\mathbf{v} \in \mathbb{R}^{1 \times d}$;

- $4 d^{2}+14 d-8$ flops are required to compute an interpolation based on the elements of a complex vector $\mathbf{v} \in \mathbb{C}^{1 \times d}$;

The detailed expressions of $\mathcal{C}_{i \text {,alg }}$ and $\mathcal{C}_{r, \text { alg }}$ are provided below for both SFE and CSFE.

SFE - The cost $\mathcal{C}_{i, \mathrm{SFE}}$ is evaluated as

$$
\mathcal{C}_{i, \mathrm{SFE}} \triangleq \mathcal{C}_{\mathbf{X}_{0}}+\mathcal{C}_{\hat{\alpha}}+\mathcal{C}_{K_{p}}+\mathcal{C}_{X_{k, \hat{\alpha}}}+\mathcal{C}_{\hat{\Delta}}
$$

where: a) $\mathcal{C}_{\mathbf{X}_{0}}=8 N_{0} \log _{2} N_{0}$ is the contribution due to the computation of the vector $\mathbf{X}_{0}$ (42); b) $\mathcal{C}_{\hat{\alpha}}=4 N_{0}$ is the contribution due to the computation of $\hat{\alpha}$ on the basis of eq. (77); c) $\mathcal{C}_{K_{p}}=6 \log _{2} N+151$ is the contribution due to the evaluation of the quantities $\left\{K_{1}(2 \hat{\alpha}), K_{2}(2 \hat{\alpha}), K_{3}(2 \hat{\alpha})\right\}$ on the basis of eq. (62); d) $\mathcal{C}_{X_{k, \hat{\alpha}}}=14 N+10$ is the contribution due to computation of $\bar{X}_{k, \hat{\alpha}}$ on the basis of eq. (63); e) $\mathcal{C}_{\hat{\Delta}}=65$ is the contribution due to the computation of the coefficients of the quadratic equation (69) and to the evaluation of its solution $\hat{\Delta}$. The cost $\mathcal{C}_{r, \mathrm{SFE}}$, instead, is evaluated as

$$
\mathcal{C}_{r, \mathrm{SFE}} \triangleq \mathcal{C}_{g}+\mathcal{C}_{\bar{X}}+\mathcal{C}_{\hat{\rho}}+\mathcal{C}_{\hat{C}}+\mathcal{C}_{X_{k, \hat{\rho}}}+\mathcal{C}_{K_{p}}+\mathcal{C}_{\hat{\Delta}}
$$

where: a) $\mathcal{C}_{g}=15$ is the contribution due to the computation of $g(\hat{F})$ on the basis of eq. (50); b) $\mathcal{C}_{\bar{X}}=6 N+4$ is the contribution due to the computation of $\bar{X}(\hat{F})$ on the basis of eq. $(38) ;$ c) $\mathcal{C}_{\hat{\rho}}=1$ is due to the evaluation of $\hat{\rho}^{(i-1)}$ on the basis of eq. (80); d) $\mathcal{C}_{\hat{C}}=17$ is the contribution due to the evaluation of the complex amplitude $\hat{C}$ on the basis of eq. (49); e) $\mathcal{C}_{X_{k, \hat{\rho}}}=14 N+10$ is the contribution due to the evaluation of the quantities $\left\{\bar{X}_{k, \hat{\rho}^{(i-1)}}\right\}$ on the basis of eq. (63); f) the cost $\mathcal{C}_{K_{p}}=6 \log _{2} N+151$ is the contribution due to the evaluation of the quantities $\left\{K_{p}\left(2 \hat{\rho}^{(i-1)}\right)\right\}$ on the basis of eq. (62) g) $\mathcal{C}_{\hat{\Delta}}=65$ is the contribution due to the computation of the coefficients of the quadratic equation (69) and to the evaluation of its solution $\hat{\Delta}$. It is worth mentioning that, as explained in Paragraph III-A, an interpolation technique can be used to compute the quantities $\bar{X}(\hat{F})$ and $\left\{\bar{X}_{k, \hat{\rho}^{(i-1)}}\right\}$. In this case, $\mathcal{C}_{\bar{X}}=4 I^{2}+14 I-8$ flops and $\mathcal{C}_{X_{k, \hat{\rho}}}=3\left(4 I^{2}+14 I-8\right)$ flops are needed for the computation of $\bar{X}(\hat{F})$ and $\left\{\bar{X}_{k, \hat{\rho}^{(i-1)}}\right\}$, respectively, if a barycentric interpolation technique is used [70]. Substituting the terms appearing in the RHSs of eqs. (161) and (162) with their expressions and, then, the resulting expressions in the RHS of eq. (160) yields

$$
\begin{aligned}
\mathcal{C}_{\mathrm{SFE}}= & 4 N_{0}+14 N+8 N_{0} \log _{2} N_{0}+6 \log _{2} N+226 \\
& +N_{\mathrm{SFE}}\left(20 N+6 \log _{2} N+269\right) .
\end{aligned}
$$

From the last expression, eq. (120) can be easily inferred.

CSFE - The cost $\mathcal{C}_{i, \mathrm{CSFE}}$ is evaluated as

$$
\mathcal{C}_{i, \mathrm{CSFE}} \triangleq \mathcal{C}_{\mathbf{X}_{0}}+\mathcal{C}_{\hat{\alpha}}+\mathcal{C}_{X_{k, \hat{\alpha}}}+\mathcal{C}_{\hat{\Delta}}
$$


where: a) $\mathcal{C}_{\mathbf{X}_{0}}$ and $\mathcal{C}_{\hat{\alpha}}$ have the same meaning as that already illustrated for the SFE; b) $\mathcal{C}_{X_{k, \hat{\alpha}}}=33 N+15$ is the contribution due to computation of $\bar{X}_{k, \hat{\alpha}}$ on the basis of eq. (63); b) $\mathcal{C}_{\hat{\Delta}}=29$ is the contribution due to the computation of the coefficients of the quadratic equation (69) and to the evaluation of its solution $\hat{\Delta}$. The cost $\mathcal{C}_{r, \mathrm{CSFE}}$, instead, is evaluated as

$$
\mathcal{C}_{r, \mathrm{CSFE}} \triangleq \mathcal{C}_{\bar{X}}+\mathcal{C}_{\hat{\rho}}+\mathcal{C}_{\hat{A}}+\mathcal{C}_{X_{k, \hat{\rho}}}+\mathcal{C}_{\hat{\Delta}}
$$

where: a) $\mathcal{C}_{\bar{X}}$ and $\mathcal{C}_{\hat{\rho}}$ have the same meaning as that illustrated for the SFE; b) $\mathcal{C}_{\hat{A}}=6 N+2$ is the contribution due to the evaluation of $\hat{A}$ on the basis of eq. (95); c) $\mathcal{C}_{X_{k, \hat{\rho}}}=33 N+15$ is the contribution due to the evaluation of the quantities $\left\{\bar{X}_{k, \hat{\rho}^{(i-1)}}\right\}$ on the basis of eq. (63); d) $\mathcal{C}_{\hat{\Delta}}=29$ is the contribution due to the computation of the coefficients of the quadratic equation (69) and to the evaluation of its solution $\hat{\Delta}$. Similarly as the SFE, an interpolation technique can be employed to compute the quantities $\bar{X}(\hat{F})$ and $\left\{\bar{X}_{k, \hat{\rho}^{(i-1)}}\right\}$. In this case, $\mathcal{C}_{\bar{X}}=4 I^{2}+14 I-8$ flops and $\mathcal{C}_{X_{k, \hat{\rho}}}=3\left(4 I^{2}+14 I-8\right)$ flops are needed for the computation of $\bar{X}(\hat{F})$ and $\bar{X}_{k, \hat{\rho}^{(i-1)}}$, respectively, if a barycentric interpolation technique is used. Substituting the terms appearing in the RHSs of eqs. (164) and (165) with their expressions and, then, the resulting expressions in the RHS of eq. (160) gives

$$
\begin{aligned}
\mathcal{C}_{\mathrm{CSFE}}= & 4 N_{0}+8 N_{0} \log _{2} N_{0}+33 N+44 \\
& +N_{\mathrm{CSFE}}(39 N+49) .
\end{aligned}
$$

From the last expression, eq. (121) can be easily inferred. It is worth noting that eqs. (160), (164), (165) and (166) are still valid for the CSDE algorithm.

\section{APPENDIX D}

In this Appendix, the bias of the CSFE algorithm is analysed in the case in which the parameter $\Delta$ is computed on the basis of eq. (74). To begin, let us assume that: a) the complex sequence $\left\{x_{c, n}\right\}$ is not affected by noise, so that (see eqs. (89) and (55))

$$
x_{c, n}=A \exp (j 2 \pi n F)=A \exp \left(j 2 \pi n\left(F_{c}+\delta F_{\mathrm{DFT}}\right)\right) ;
$$

b) $\hat{\alpha}=\alpha$ (see eqs. (76) and (77)). Then, it is easy to show that (see eqs. (63) and (64))

$$
\bar{X}_{1, \rho^{(i-1)}}=\frac{A}{N_{0}} \sum_{n=0}^{N-1} n \exp (j 2 \pi n \vartheta)
$$

and

$$
\bar{X}_{2, \rho^{(i-1)}}=\frac{A}{N_{0}} \sum_{n=0}^{N-1} n^{2} \exp (j 2 \pi n \vartheta)
$$

where $\vartheta_{i} \triangleq\left(\delta-\hat{\delta}_{i}\right) F_{\mathrm{DFT}}$ and $\hat{\delta}_{i} \triangleq\left(\hat{F}^{(i)}-\hat{F}_{c}^{(0)}\right) / F_{\mathrm{DFT}}$. The identities (84) and (85) allow us to rewrite eqs. (168) and (169) as

$$
\bar{X}_{1, \rho^{(i-1)}}=\frac{1}{N_{0}} A \frac{(N-1) q_{i}^{N+1}-N q_{i}^{N}+q_{i}}{\left(q_{i}-1\right)^{2}}
$$

and

$$
\bar{X}_{2, \rho^{(i-1)}}=\frac{1}{N_{0}} A \frac{(N-1)^{2} q_{i}^{N+2}+\left(-2 N^{2}+2 N+1\right) q_{i}^{N+1}+N^{2} q_{i}^{N}-q_{i}^{2}-q_{i}}{\left(q_{i}-1\right)^{3}}
$$

respectively, where $q_{i} \triangleq \exp \left(j 2 \pi \vartheta_{i}\right)$. If the normalised frequency $F_{\mathrm{DFT}}$ (41) is small enough (i.e., if the FFT order $N_{0}$ is large enough), the quantity $q_{i}$ can be approximated through its truncated Taylor series $1+j 2 \pi \vartheta_{i}-2 \pi^{2} \vartheta_{i}^{2}-j 4 \pi^{3} \vartheta_{i}^{3} / 3$. Then, substituting this approximation in the RHS of eqs. (170) and (171) and, then, the resulting expressions in the RHSs of eqs. (99) and (100) yields, after some manipulation,

$$
b\left(\rho^{(i-1)}\right) \triangleq \Re\left\{\hat{A}^{*} \bar{X}_{2, \rho^{(i-1)}}\right\}=F_{\mathrm{DFT}} \frac{|A|^{2}}{6}(2 N-1) N(N-1) .
$$

and

$$
c\left(\rho^{(i-1)}\right) \triangleq-\Im\left\{\hat{A}^{*} \bar{X}_{1, \rho^{(i-1)}}\right\}=-F_{\mathrm{DFT}}|A|^{2} \frac{\pi}{3} \frac{(2 N-1) N(N-1) \vartheta_{i}}{1+4 \pi^{2} \vartheta_{i}^{2}},
$$

respectively. Then, substituting the RHSs of the last two equations in that of eq. (74) gives

$$
\hat{\Delta}^{(i)}=\frac{2 \pi \vartheta_{i}}{1+4 \pi^{2} \vartheta_{i}^{2}} .
$$

Note that $\vartheta_{i}$ gets smaller as $i$ increases if the CSFE converges; therefore, the last expression can be approximated as

$$
\hat{\Delta}^{(i)} \simeq 2 \pi \vartheta_{i}=2 \pi\left(\delta-\hat{\delta}_{i}\right) F_{\mathrm{DFT}}=\Delta-\hat{\Delta}_{i}
$$

where $\hat{\Delta}_{i} \triangleq 2 \pi \hat{\delta}_{i} F_{\mathrm{DFT}}$. This proves that the CSFE is unbiased. 


\section{REFERENCES}

[1] S. M. Patole, M. Torlak, D. Wang, and M. Ali, "Automotive radars: A review of signal processing techniques," IEEE Signal Process. Mag., vol. 34, no. 2, pp. 22-35, Mar. 2017.

[2] T. Schmidl and D. Cox, "Robust frequency and timing synchronization for OFDM," IEEE Trans. Commun., vol. 45, no. 12, pp. 1613-1621, Dec. 1997.

[3] P. Whittle, "The simultaneous estimation of a time series harmonic components and covariance structure," p. 15, 1952.

[4] A. M. Walker, "On the Estimation of a Harmonic Component in a Time Series with Stationary Independent Residuals," Biometrika, vol. 58, no. 1, pp. 21-36, 1971.

[5] D. C. Rife and R. R. Boorstyn, "Multiple tone parameter estimation from discrete-time observations," Bell Syst. Tech. J., vol. 55, no. 9, pp. 1389-1410, Nov. 1976.

[6] S. Verdú, Multiuser Detection. Cambridge University Press, 1998.

[7] J. A. Hogbom, "Aperture Synthesis with Non-Rectangular Distribution of Interferometric Baselines," Astron Astrophys Suppl, vol. 15, no. 3, pp. 417-426, Jun. 1974.

[8] J. Tsao and B. Steinberg, "Reduction of sidelobe and speckle artifacts in microwave imaging: The CLEAN technique," IEEE Trans. Antennas Propag., vol. 36, no. 4, pp. 543-556, Apr. 1988.

[9] D. Zankl, S. Schuster, R. Feger, and A. Stelzer, "What a Blast!: A Massive MIMO Radar System for Monitoring the Surface in Steel Industry Blast Furnaces," IEEE Microw. Mag., vol. 18, no. 6, pp. 52-69, Sep. 2017.

[10] E. Sirignano, A. Davoli, G. M. Vitetta, and F. Viappiani, "A Comparative Analysis of Deterministic Detection and Estimation Techniques for MIMO SFCW Radars," IEEE Access, vol. 7, pp. 129848-129861, 2019.

[11] M. Macleod, "Fast nearly ML estimation of the parameters of real or complex single tones or resolved multiple tones," IEEE Trans. Signal Process., vol. 46, no. 1 , pp. 141-148, Jan. 1998.

[12] R. Schmidt, "Multiple emitter location and signal parameter estimation," IEEE Trans. Antennas Propag., vol. 34, no. 3, pp. 276-280, Mar. 1986.

[13] R. Roy and T. Kailath, "ESPRIT-estimation of signal parameters via rotational invariance techniques," IEEE Trans. Acoust. Speech Signal Process., vol. 37, no. 7, pp. 984-995, Jul. 1989.

[14] G. Xu and T. Kailath, "Fast subspace decomposition," IEEE Trans. Signal Process., vol. 42, no. 3, pp. 539-551, Mar. 1994.

[15] J. Xin and A. Sano, "Computationally efficient subspace-based method for direction-of-arrival estimation without eigendecomposition," IEEE Trans. Signal Process., vol. 52, no. 4, pp. 876-893, Apr. 2004.

[16] J. Capon, "High-Resolution Frequency-Wavenumber Spectrum Analysis," Proc. IEEE, vol. 57, no. 8, pp. 1408-1418, Aug. 1969.

[17] J. Li and P. Stoica, "An Adaptive Filtering Approach to Spectral Estimation and SAR Imaging," IEEE Trans. Signal Process., vol. 44, no. 6, pp. 1469-1484, Jun. 1996.

[18] T. Yardibi, J. Li, P. Stoica, M. Xue, and A. B. Baggeroer, "Source Localization and Sensing: A Nonparametric Iterative Adaptive Approach Based on Weighted Least Squares," IEEE Trans. Aerosp. Electron. Syst., vol. 46, no. 1, pp. 425-443, Jan. 2010.

[19] G.-O. Glentis, "A Fast Algorithm for APES and Capon Spectral Estimation," IEEE Trans. Signal Process., vol. 56, no. 9, pp. 4207-4220, Sep. 2008.

[20] K. Angelopoulos, G. O. Glentis, and A. Jakobsson, "Computationally Efficient Capon- and APES-Based Coherence Spectrum Estimation," IEEE Trans. Signal Process., vol. 60, no. 12, pp. 6674-6681, Dec. 2012.

[21] P. Gough, "A Fast Spectral Estimation Algorithm Based on the FFT," IEEE Trans. Signal Process., vol. 42, no. 6, pp. 1317-1322, Jun. 1994.

[22] J. Li and P. Stoica, "Efficient Mixed-Spectrum Estimation with Applications to Target Feature Extraction," IEEE Trans. Signal Process., vol. 44, no. 2, pp. 281-295, Feb. 1996.

[23] J. Rissanen, "A Universal Prior for Integers and Estimation by Minimum Description Length," Ann. Stat., vol. 11, no. 2, pp. 416-431, Jun. 1983.

[24] D. C. Rife and G. A. Vincent, "Use of the Discrete Fourier Transform in the Measurement of Frequencies and Levels of Tones," Bell Syst. Tech. J., vol. 49, no. 2, pp. 197-228, Feb. 1970.

[25] B. Quinn, "Estimating frequency by interpolation using Fourier coefficients," IEEE Trans. Signal Process., vol. 42, no. 5, pp. 1264-1268, May 1994.

[26] _ _ "Estimation of frequency, amplitude, and phase from the DFT of a time series," IEEE Trans. Signal Process., vol. 45, no. 3, pp. 814-817, Mar. 1997.

[27] E. Jacobsen and P. Kootsookos, "Fast, Accurate Frequency Estimators [DSP Tips Tricks]," IEEE Signal Process. Mag., vol. 24, no. 3, pp. 123-125, May 2007.

[28] Ç. Candan, "A Method For Fine Resolution Frequency Estimation From Three DFT Samples," IEEE Signal Process. Lett., vol. 18, no. 6, pp. 351-354, Jun. 2011.

[29] — " "Analysis and Further Improvement of Fine Resolution Frequency Estimation Method From Three DFT Samples," IEEE Signal Process. Lett., vol. 20, no. 9, pp. 913-916, Sep. 2013.

[30] U. Orguner and Ç. Candan, "A fine-resolution frequency estimator using an arbitrary number of DFT coefficients," Signal Processing, vol. 105, pp. 17-21, Dec. 2014.

[31] C. Yang and G. Wei, "A Noniterative Frequency Estimator With Rational Combination of Three Spectrum Lines," IEEE Trans. Signal Process., vol. 59, no. 10, pp. 5065-5070, Oct. 2011.

[32] X. Liang, A. Liu, X. Pan, Q. Zhang, and F. Chen, “A New and Accurate Estimator With Analytical Expression for Frequency Estimation,” IEEE Commun. Lett., vol. 20, no. 1, pp. 105-108, Jan. 2016.

[33] L. Fan and G. Qi, "Frequency Estimator of Sinusoid Based on Interpolation of Three DFT Spectral Lines," Signal Processing, vol. 144, pp. 52-60, Mar. 2018.

[34] L. Fan, G. Qi, J. Xing, J. Jin, J. Liu, and Z. Wang, “Accurate Frequency Estimator of Sinusoid Based on Interpolation of FFT and DTFT," IEEE Access, vol. 8, pp. 44373-44380, 2020.

[35] D. Rife and R. Boorstyn, "Single tone parameter estimation from discrete-time observations," IEEE Trans. Inf. Theory, vol. 20, no. 5, pp. 591-598, Sep. 1974.

[36] T. Abatzoglou, "A Fast Maximum Likelihood Algorithm for Frequency Estimation of a Sinusoid Based on Newton’s Method," IEEE Trans. Acoust. Speech Signal Process., vol. 33, no. 1, pp. 77-89, Feb. 1985.

[37] Y. V. Zakharov and T. C. Tozer, "Frequency estimator with dichotomous search of periodogram peak," Electron. Lett., vol. 35, no. 19, pp. 1608-1609, Sep. 1999.

[38] E. Aboutanios, "A Modified Dichotomous Search Frequency Estimator," IEEE Signal Process. Lett., vol. 11, no. 2, pp. $186-188$, Feb. 2004.

[39] S. Djukanović and I. Djurović, "Robust M-Periodogram with Dichotomous Search," Signal Processing, vol. 91, no. 10, pp. 2410-2414, Oct. 2011.

[40] E. Aboutanios and B. Mulgrew, "Iterative Frequency Estimation by Interpolation on Fourier Coefficients," IEEE Trans. Signal Process., vol. 53, no. 4, pp. 1237-1242, Apr. 2005.

[41] E. Aboutanios, "Estimating the Parameters of Sinusoids and Decaying Sinusoids in Noise," IEEE Instrum. Meas. Mag., vol. 14, no. 2, pp. 8-14, Apr. 2011.

[42] S. Minhas and E. Aboutanios, "Estimation of the frequency of a complex exponential," in Proc. of the 2008 IEEE International Conference on Acoustics, Speech and Signal Processing, Mar. 2008, pp. 3693-3696.

[43] Y. Liu, "Generalization of iterative Fourier interpolation algorithms for single frequency estimation," Digit. Signal Process., p. 9, 2011.

[44] I. Djurovic, "Estimation of the Sinusoidal Signal Frequency Based on the Marginal Median DFT," IEEE Trans. Signal Process., vol. 55, no. 5, pp. 2043-2051, May 2007. 
[45] J.-R. Liao and C.-M. Chen, "Analysis and reduction of estimation bias for an iterative frequency estimator of complex sinusoid," in Proc. of the 2013 IEEE International Conference on Acoustics, Speech and Signal Processing, May 2013, pp. 6138-6142.

[46] J.-R. Liao and S. Lo, “Analytical solutions for frequency estimators by interpolation of DFT coefficients," Signal Processing, vol. 100, pp. 93-100, Jul. 2014.

[47] Y. V. Zakharov, V. M. Baronkin, and T. C. Tozer, "DFT-based frequency estimators with narrow acquisition range," IEE Proc. - Commun., vol. 148, no. 1, pp. 1-7, Feb. 2001.

[48] C.-F. Huang, H.-P. Lu, and W.-H. Chieng, "Estimation of Single-Tone Signal Frequency with Special Reference to a Frequency-Modulated Continuous Wave System," Meas. Sci. Technol., vol. 23, no. 3, Mar. 2012.

[49] S. Ye and E. Aboutanios, "An algorithm for the parameter estimation of multiple superimposed exponentials in noise," in Proc. of the 2015 IEEE International Conference on Acoustics, Speech and Signal Processing (ICASSP), Apr. 2015, pp. 3457-3461.

[50] _ "Rapid accurate frequency estimation of multiple resolved exponentials in noise," Signal Processing, vol. 132, pp. 29-39, Mar. 2017.

[51] A. Serbes and K. Qaraqe, “A Fast Method for Estimating Frequencies of Multiple Sinusoidals,” IEEE Signal Process. Lett., vol. 27, pp. 386-390, 2020.

[52] A. Serbes, "Fast and Efficient Sinusoidal Frequency Estimation by Using the DFT Coefficients," IEEE Trans. Commun., vol. 67, no. 3, pp. 2333-2342, Mar. 2019.

[53] T. Grandke, "Interpolation Algorithms for Discrete Fourier Transforms of Weighted Signals," IEEE Trans. Instrum. Meas., vol. 32, no. 2, pp. 350-355, Jun. 1983.

[54] M. Macleod, "Fast DFT-Domain Algorithms for Near-Optimal Tonal Detection and Frequency Estimation,” Institute of Acoustics Proceedings, vol. 13, pp. 102-109, 1991.

[55] D. Belega and D. Petri, "Sine-Wave Parameter Estimation by Interpolated DFT Method Based on New Cosine Windows with High Interference Rejection Capability," Digital Signal Processing, vol. 33, pp. 60-70, Oct. 2014.

[56] K. Duda, L. B. Magalas, M. Majewski, and T. P. Zielinski, "DFT-based Estimation of Damped Oscillation Parameters in Low-Frequency Mechanical Spectroscopy," IEEE Trans. Instrum. Meas., vol. 60, no. 11, pp. 3608-3618, Nov. 2011.

[57] M. Feder and E. Weinstein, "Parameter estimation of superimposed signals using the EM algorithm," IEEE Trans. Acoust. Speech Signal Process., vol. 36, no. 4, pp. 477-489, Apr. 1988.

[58] J. Fessler and A. Hero, "Space-Alternating Generalized Expectation-Maximization Algorithm,” IEEE Trans. Signal Process., vol. 42, no. 10, pp. 26642677, Oct. 1994.

[59] B. Fleury, M. Tschudin, R. Heddergott, D. Dahlhaus, and K. Ingeman Pedersen, "Channel Parameter Estimation in Mobile Radio Environments Using the SAGE Algorithm,” IEEE J. Sel. Areas Commun., vol. 17, no. 3, pp. 434-450, Mar. 1999.

[60] P. Bloomfield, Fourier Analysis of Time Series: An Introduction. New York: Wiley, 1976.

[61] J. Li and P. Stoica, "MIMO Radar with Colocated Antennas," IEEE Signal Process. Mag., vol. 24, no. 5, pp. 106-114, Sep. 2007.

[62] M. A. Richards, Fundamentals of Radar Signal Processing. New York: McGraw-Hill, 2005.

[63] I. Ziskind and M. Wax, "Maximum likelihood localization of multiple sources by alternating projection," IEEE Trans. Acoust. Speech Signal Process., vol. 36, no. 10, pp. 1553-1560, Oct. 1988.

[64] J. Li and P. Stoica, Eds., MIMO Radar Signal Processing. Hoboken, NJ: J. Wiley \& Sons, 2009.

[65] J. Gamba, Radar Signal Processing for Autonomous Driving. Springer Singapore, 2020.

[66] L. L. Scharf and C. Demeure, Statistical Signal Processing: Detection, Estimation, and Time Series Analysis. Addison-Wesley Pub. Co, 1991.

[67] I. Bilik, O. Longman, S. Villeval, and J. Tabrikian, "The Rise of Radar for Autonomous Vehicles: Signal Processing Solutions and Future Research Directions," IEEE Signal Process. Mag., vol. 36, no. 5, pp. 20-31, Sep. 2019.

[68] S. Sun, A. P. Petropulu, and H. V. Poor, "MIMO Radar for Advanced Driver-Assistance Systems and Autonomous Driving: Advantages and Challenges," IEEE Signal Process. Mag., vol. 37, no. 4, pp. 98-117, Jul. 2020.

[69] A. Serbes, "Fast and Efficient Estimation of Frequencies," IEEE Trans. Commun., vol. 69, no. 6, pp. 4054-4066, Jun. 2021.

[70] J. Selva, "Efficient Wideband DOA Estimation Through Function Evaluation Techniques," IEEE Trans. Signal Process., vol. 66, no. 12, pp. 3112-3123, Jun. 2018.

[71] Y.-X. Yao and S. Pandit, "Cramer-Rao lower bounds for a damped sinusoidal process," IEEE Trans. Signal Process., vol. 43, no. 4, pp. 878-885, Apr. 1995.

[72] "Imaging radar using cascaded mmwave sensor reference design - tidep-01012." [Online]. Available: https://www.ti.com/tool/TIDEP-01012

[73] "Vito-in-car radar." [Online]. Available: https://vayyar.com/auto/.

[74] "Camboard pico flexx." [Online]. Available: https://pmdtec.com/picofamily/

[75] G. H. Golub and C. F. Van Loan, Matrix Computations, 3rd ed., ser. Johns Hopkins Studies in the Mathematical Sciences. Baltimore: Johns Hopkins University Press, 1996. 